\title{
Exploiting Chitin as a Source of Biologically Fixed Nitrogen: Formation and Full Characterization of Small Molecule Hetero- and Carbo-Cyclic Pyrolysis Products
}

Maryam Nikahd, ${ }^{\dagger}$ Jiri Mikusek, ${ }^{\dagger}$ Li-Juan Yu, ${ }^{\dagger, \S}$ Michelle L. Coote ${ }^{\dagger, \S}$ Martin G. Banwell, ${ }^{* \dagger}{ }^{\dagger}{ }^{\ddagger}$ Chenxi $\mathrm{Ma}^{\dagger}$ and Michael G. Gardiner ${ }^{\dagger}$

${ }^{\dagger}$ Research School of Chemistry, Institute of Advanced Studies The Australian National University, Canberra, ACT 2601, Australia

${ }^{\S}$ ARC Centre of Excellence for Electromaterials Science, Research School of Chemistry, The Australian National University, Canberra, ACT 2601, Australia

\footnotetext{
Institute for Advanced and Applied Chemical Synthesis, Jinan University, Guangzhou 510632, China
} 
Anisotropic Displacement Ellipsoid Plot from the Single-crystal X-ray Analysis of Compound $\mathbf{5}$

Anisotropic Displacement Ellipsoid Plot from the Single-crystal X-ray Analysis of Compound 6

Anisotropic Displacement Ellipsoid Plot from the Single-crystal $\mathrm{X}$-ray Analysis of Compound $\mathbf{8}$

Anisotropic Displacement Ellipsoid Plot from the Single-crystal X-ray Analysis of Compound 9

Anisotropic Displacement Ellipsoid Plot from the Single-crystal X-ray Analysis of Compound $\mathbf{1 0}$

Anisotropic Displacement Ellipsoid Plot from the Single-crystal X-ray Analysis of Compound 11

Anisotropic Displacement Ellipsoid Plot from the Single-crystal X-ray Analysis of Compound 12

Anisotropic Displacement Ellipsoid Plot from the Single-crystal X-ray Analysis of Compound $\mathbf{1 3}$

Anisotropic Displacement Ellipsoid Plot from the Single-crystal X-ray Analysis of Compound 14

Anisotropic Displacement Ellipsoid Plot from the Single-crystal X-ray Analysis of Compound $\mathbf{1 5}$ 


\section{Apparati Used in Pyrolysis Experiments}

Pictures of the apparati used in the pyrolyses described above are shown in Figures S1-S8.

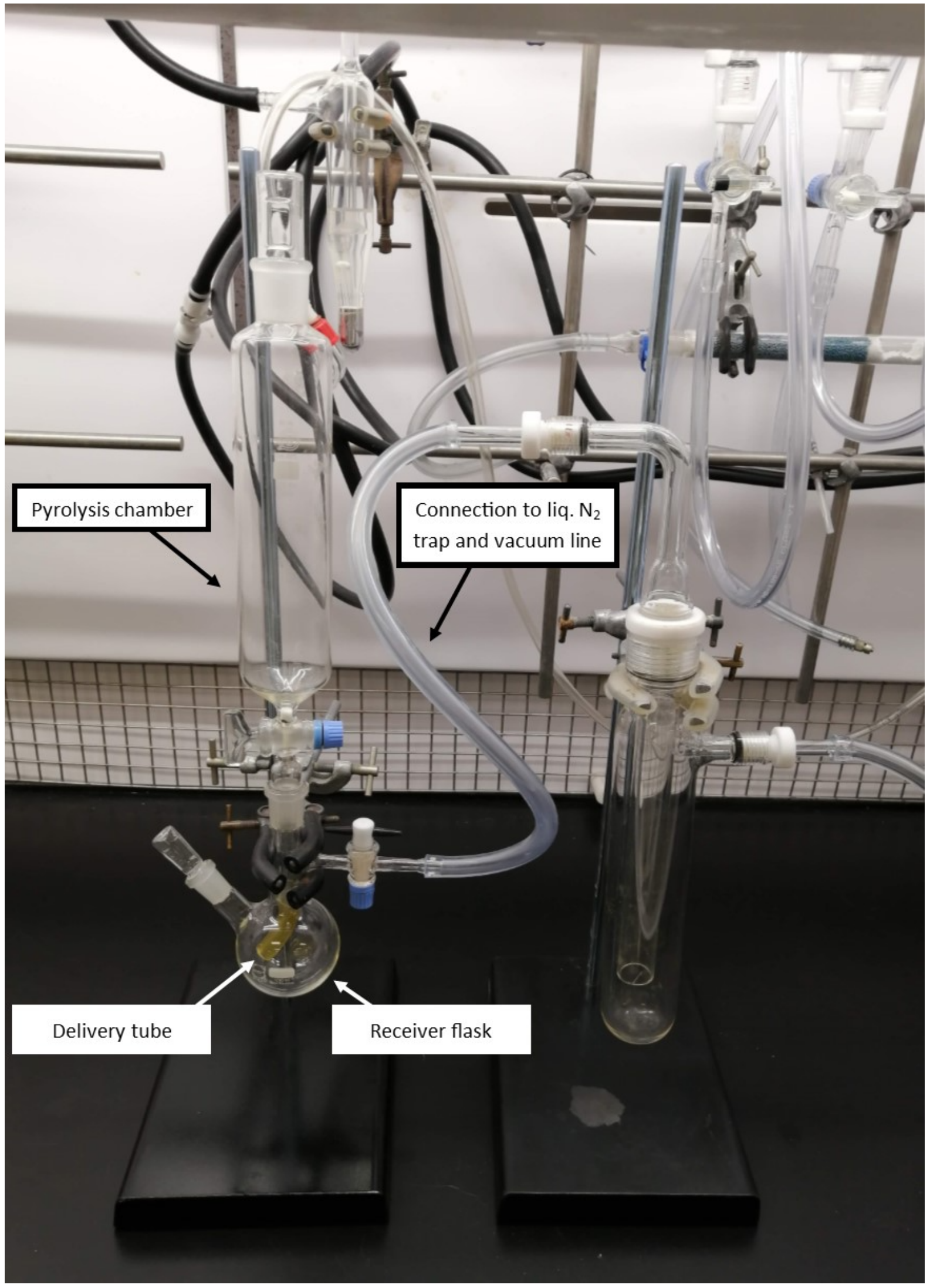

Figure S1: Empty Type 1 Pyrolysis Apparatus 


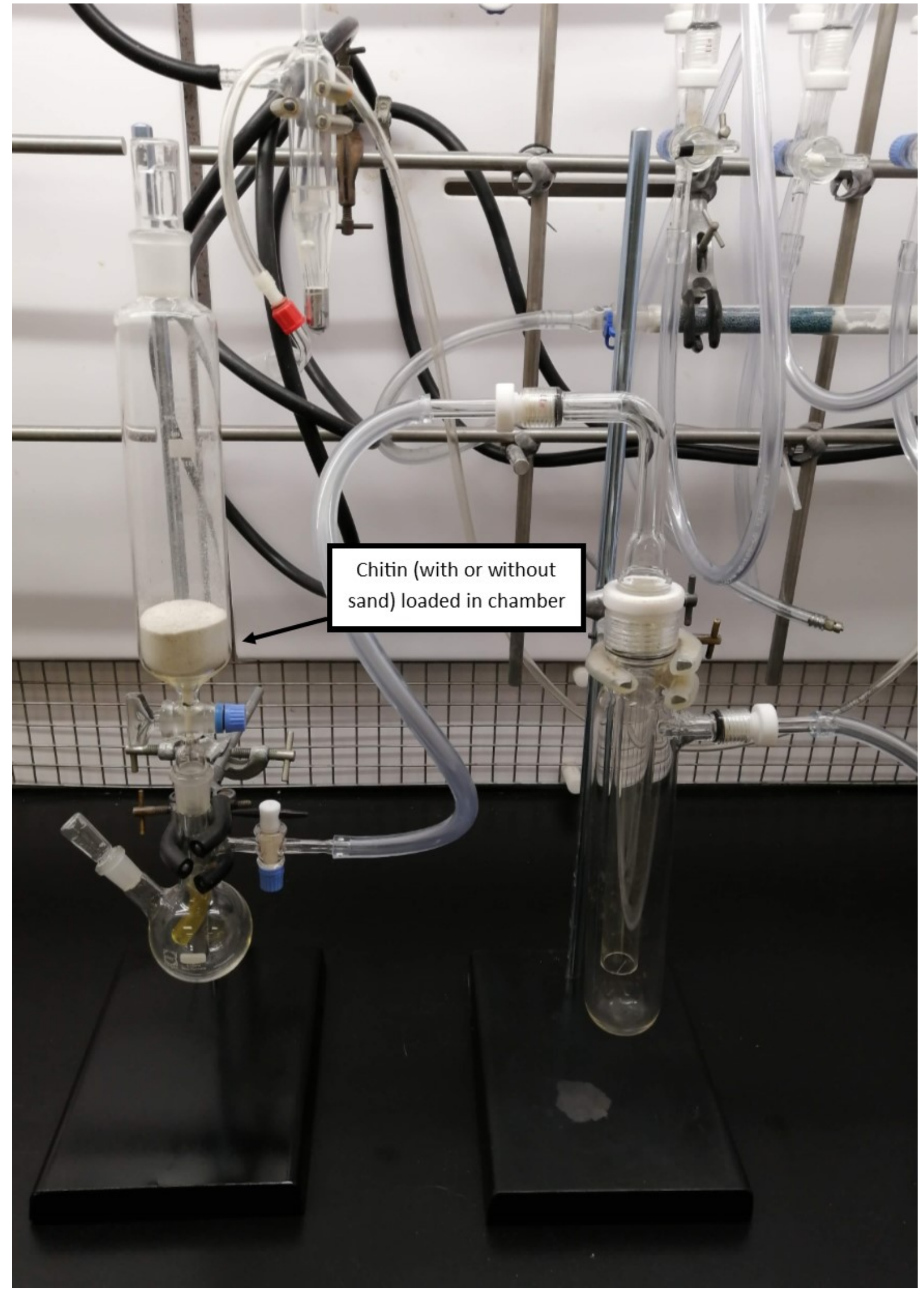

Figure S2: Type 1 Pyrolysis Apparatus Containing Substrate in Heating Chamber 


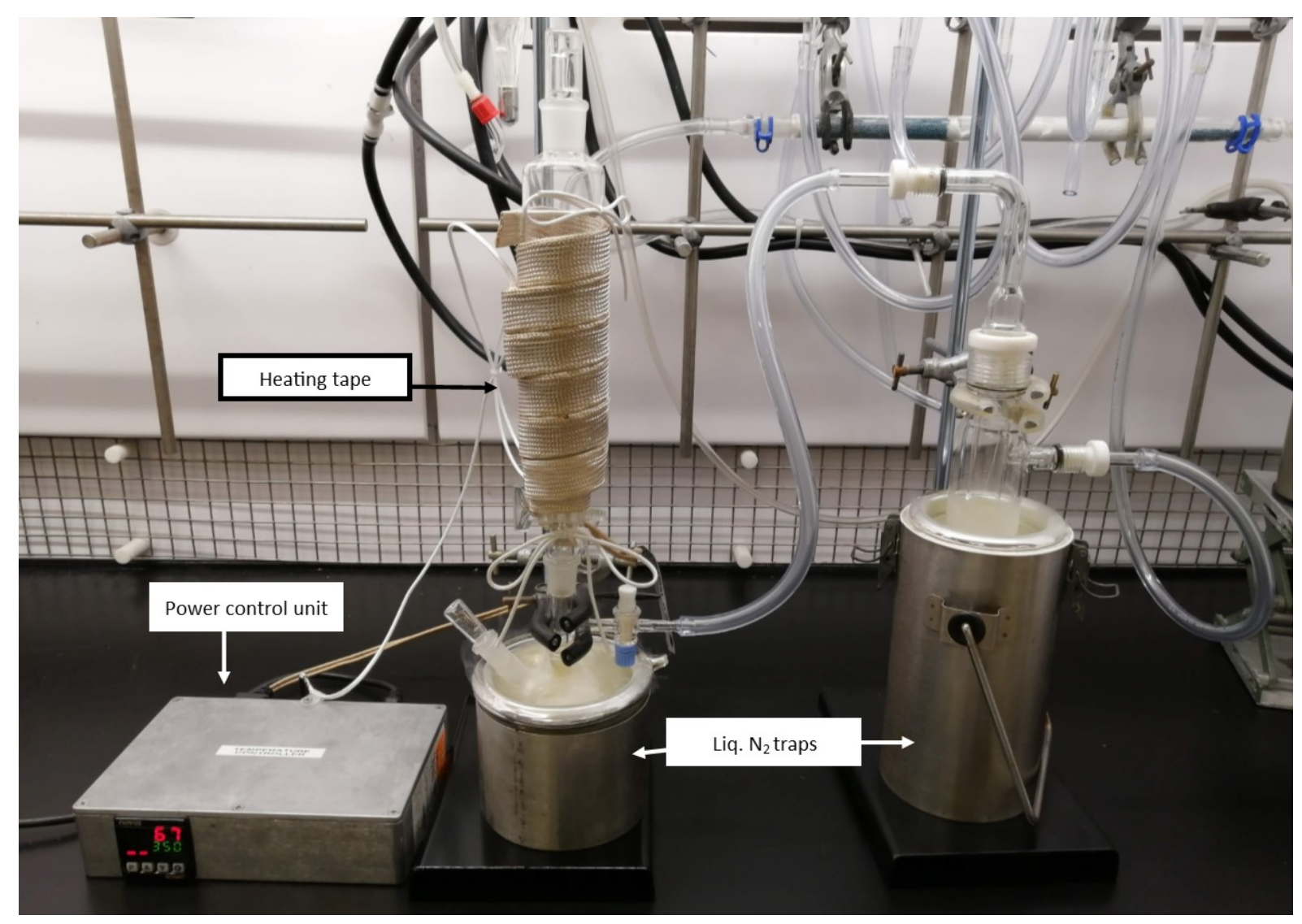

Figure S3: Charged Type 1 Pyrolysis Apparatus with Heating Chamber Wrapped with Heating Tape Connected to Control Unit and Low Temperature Traps in Place 


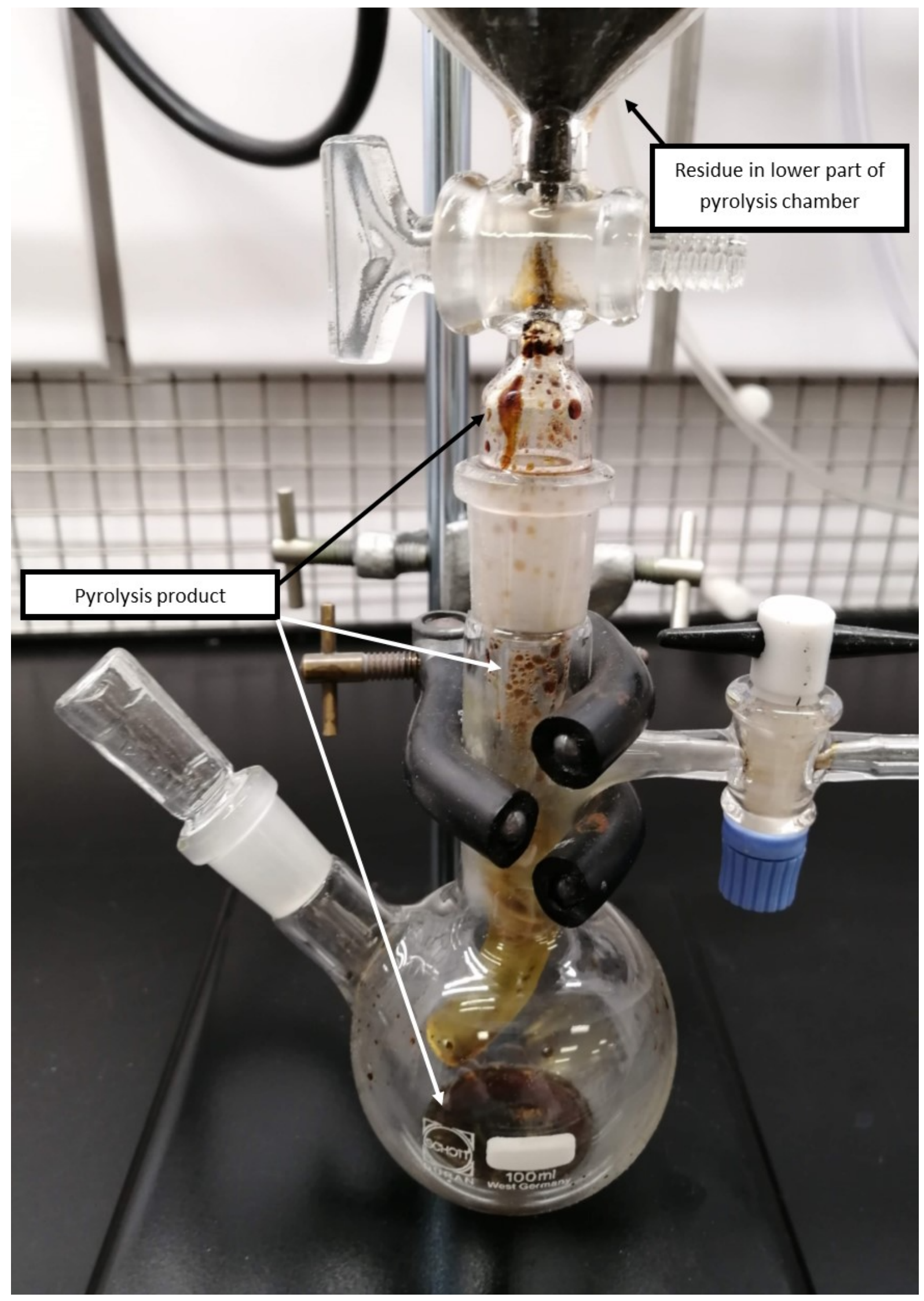

Figure S4: Close-up of the Relevant Part of Cooled Type 1 Pyrolysis Apparatus with Heating Tape Removed and Showing the Residue Obtained. 


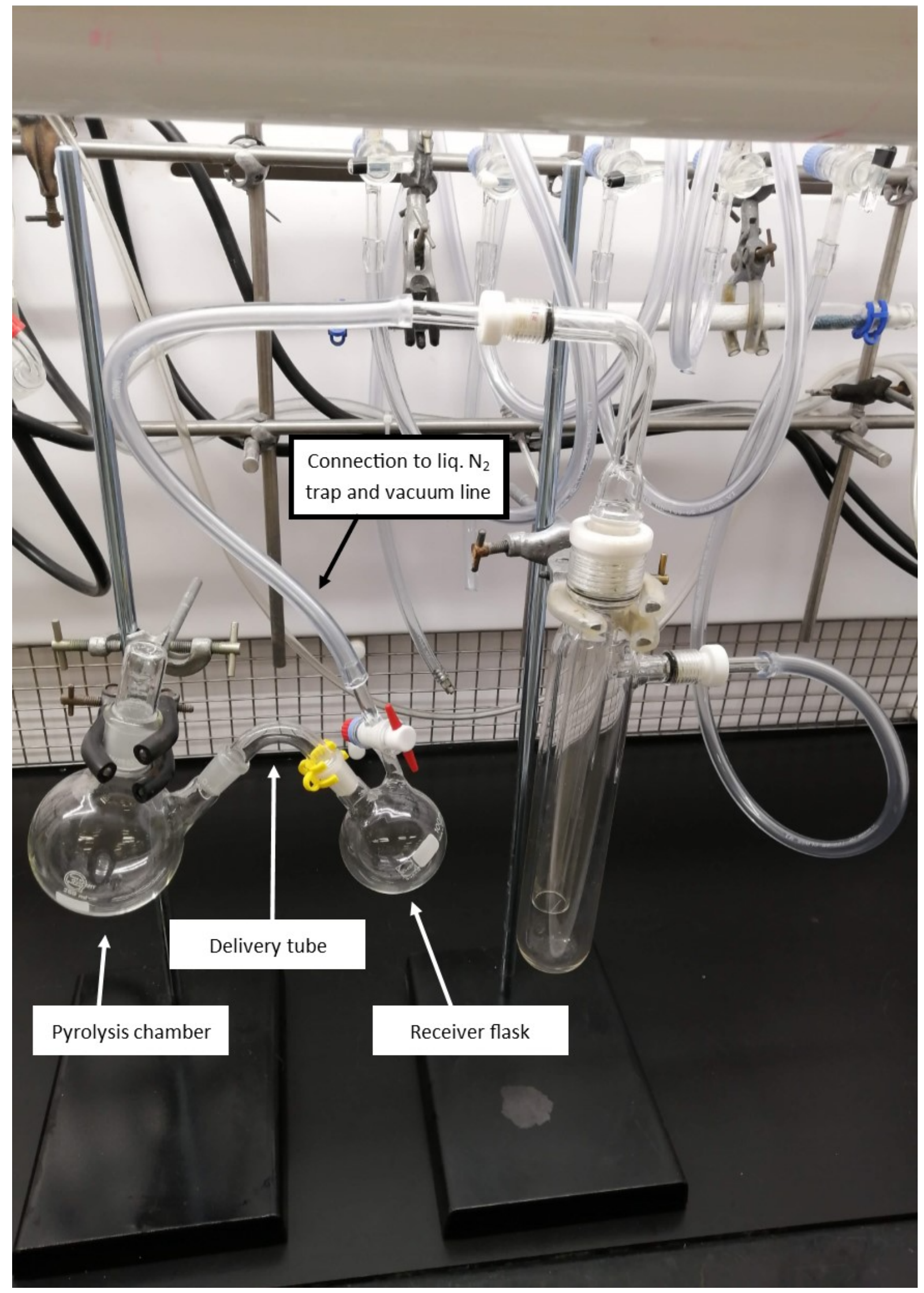

Figure S5: Empty Type 2 Pyrolysis Apparatus 


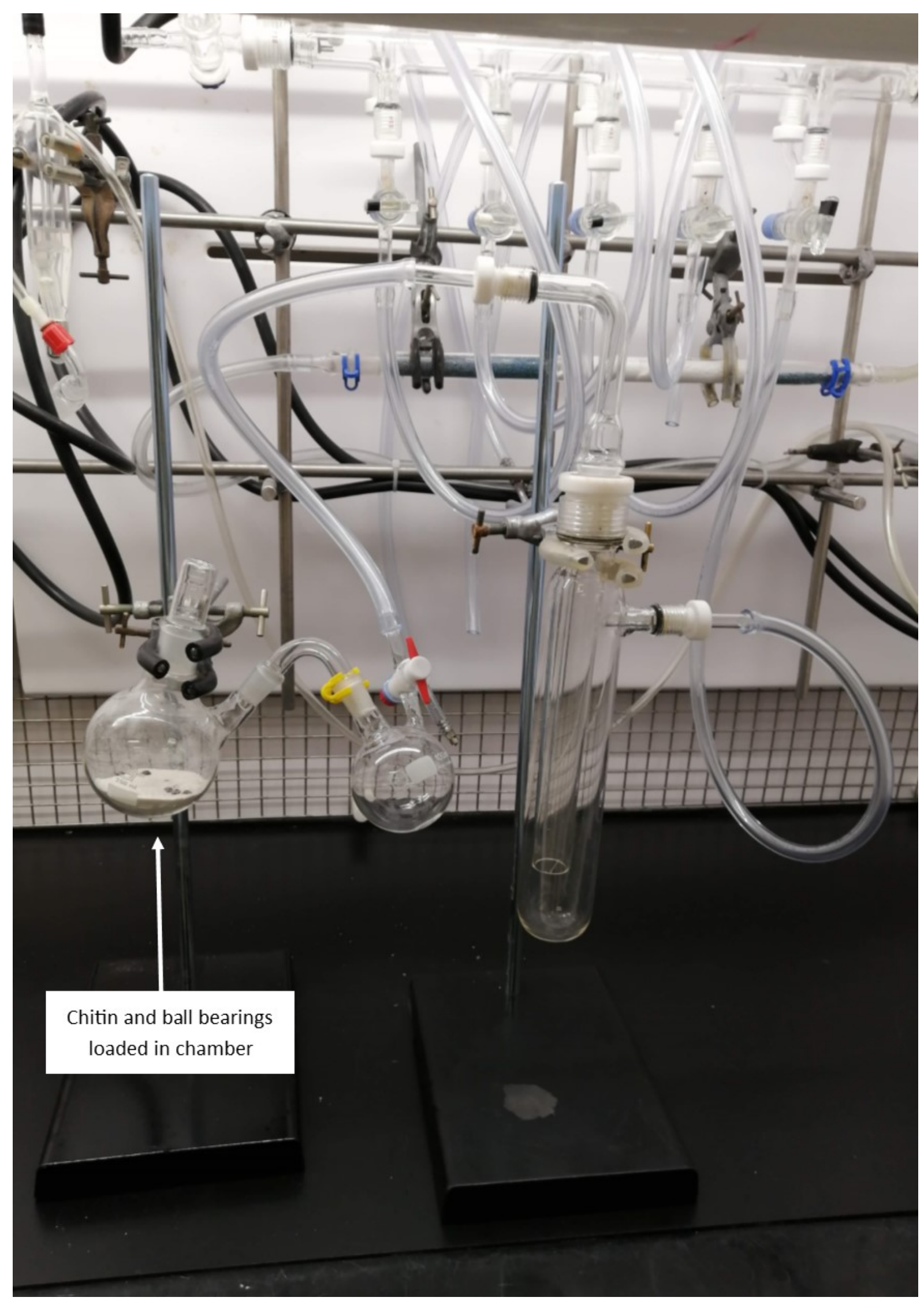

Figure S6: Type 2 Pyrolysis Apparatus Containing Substrate in Heating Chamber 


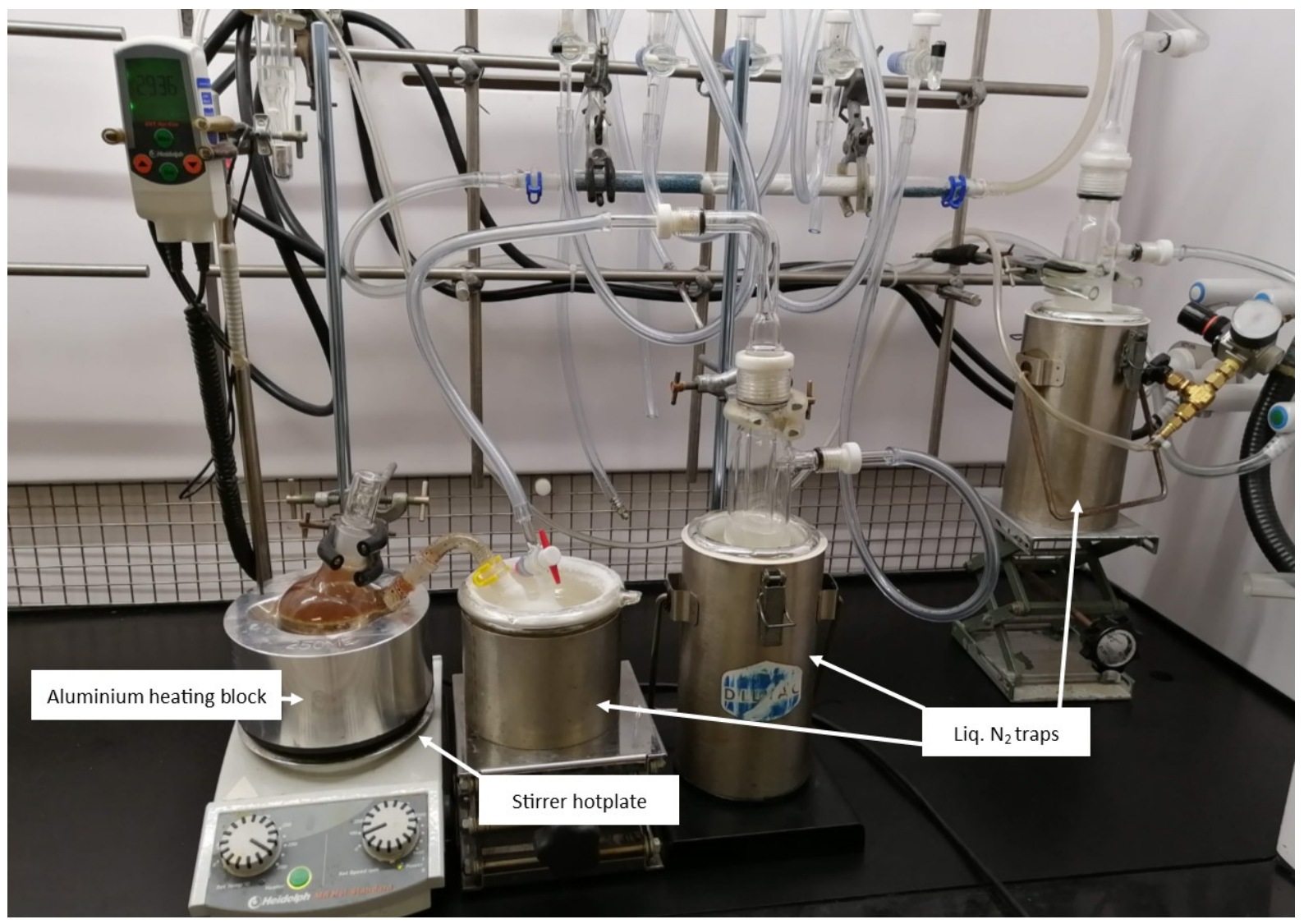

Figure S7: Charged Type 2 Pyrolysis Apparatus with Heating Block and Low Temperature Traps in Place (and pyrolysis underway). 


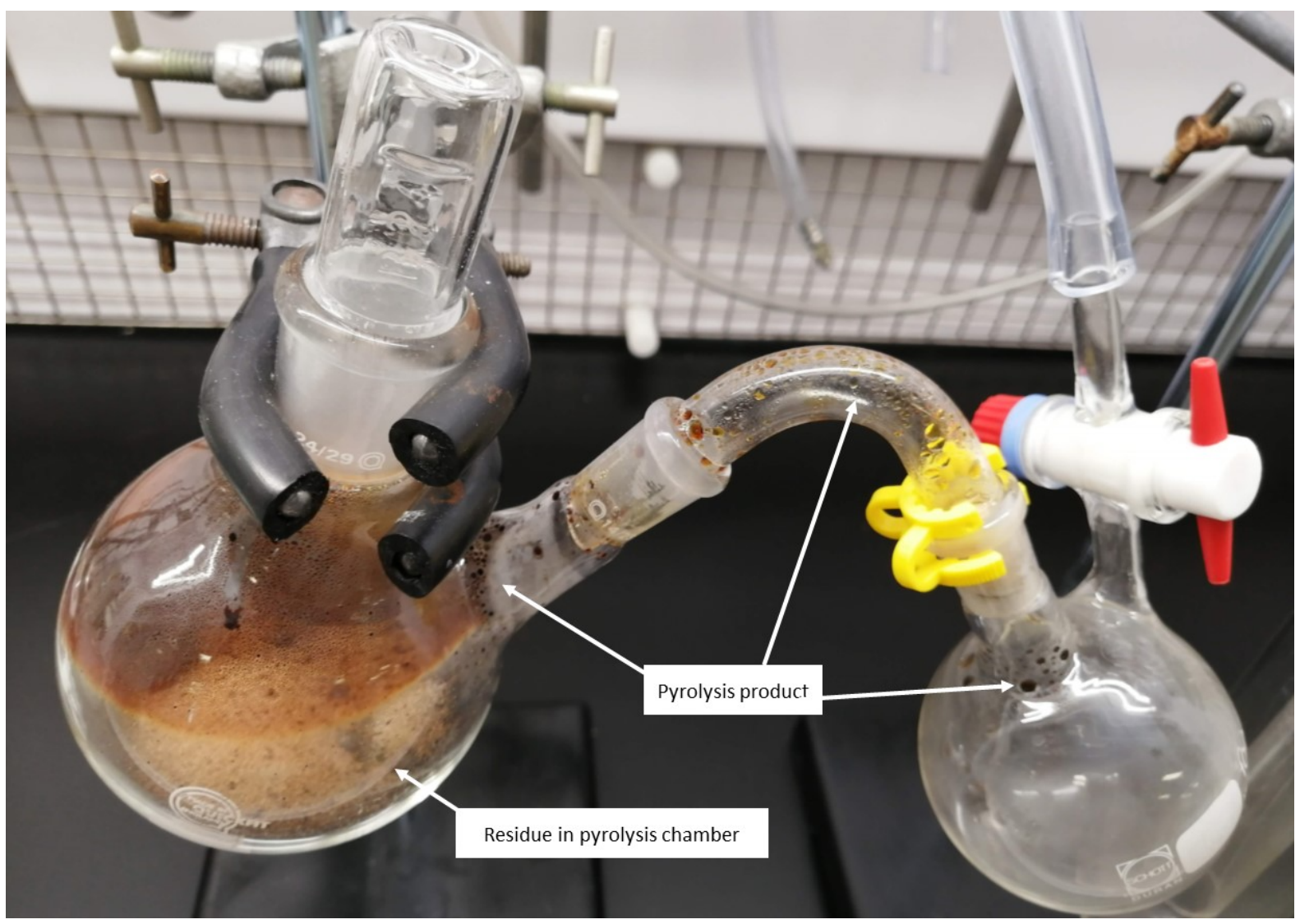

Figure S8: Cooled Type 2 Pyrolysis Apparatus with Heating Block Removed and Showing the Residue Obtained. 


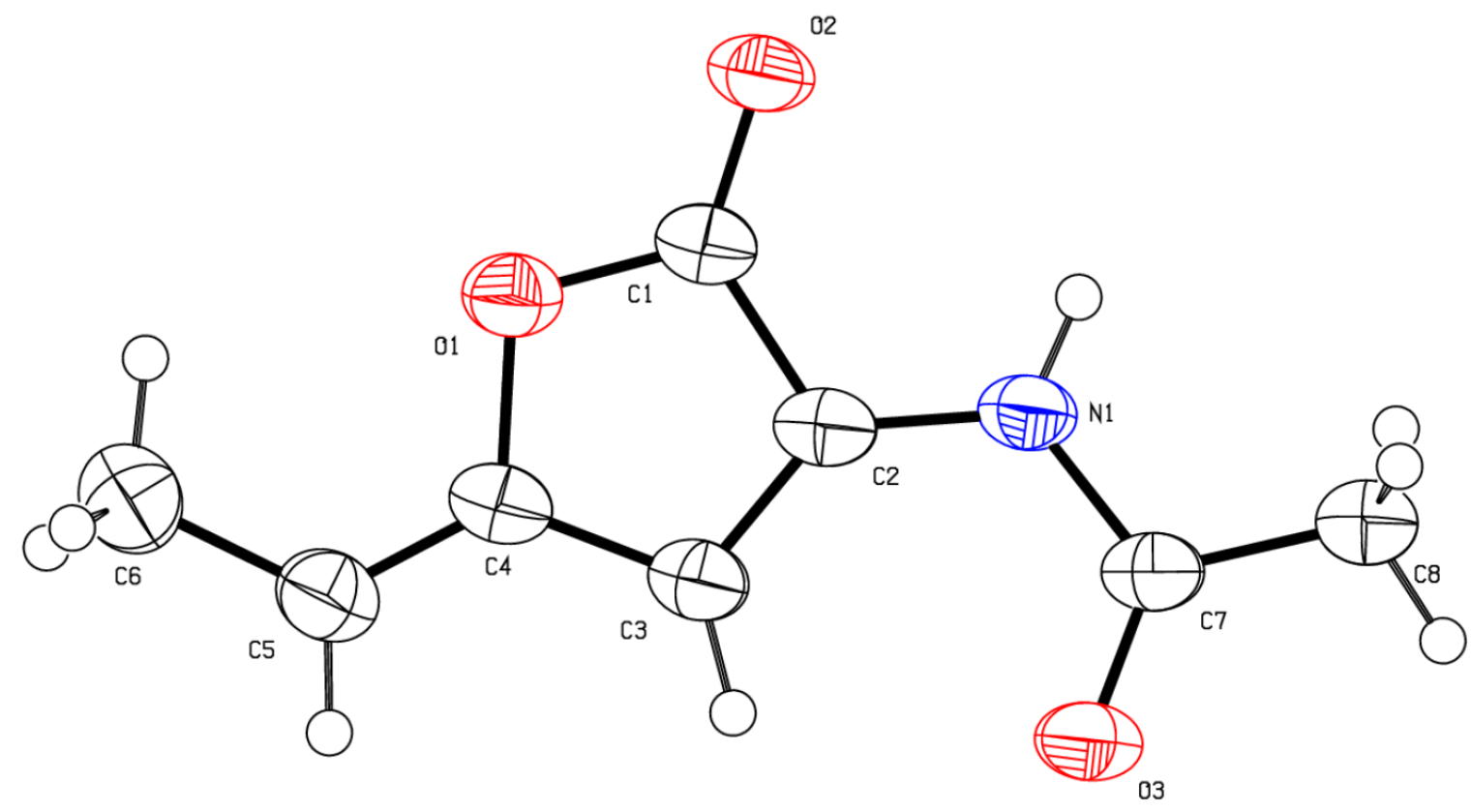

Figure S1: Structure of compound 5 (CCDC 1943032). Anisotropic displacement ellipsoids show $30 \%$ probability levels. Hydrogen atoms are drawn as circles with small radii (crystal grown from methanol). 


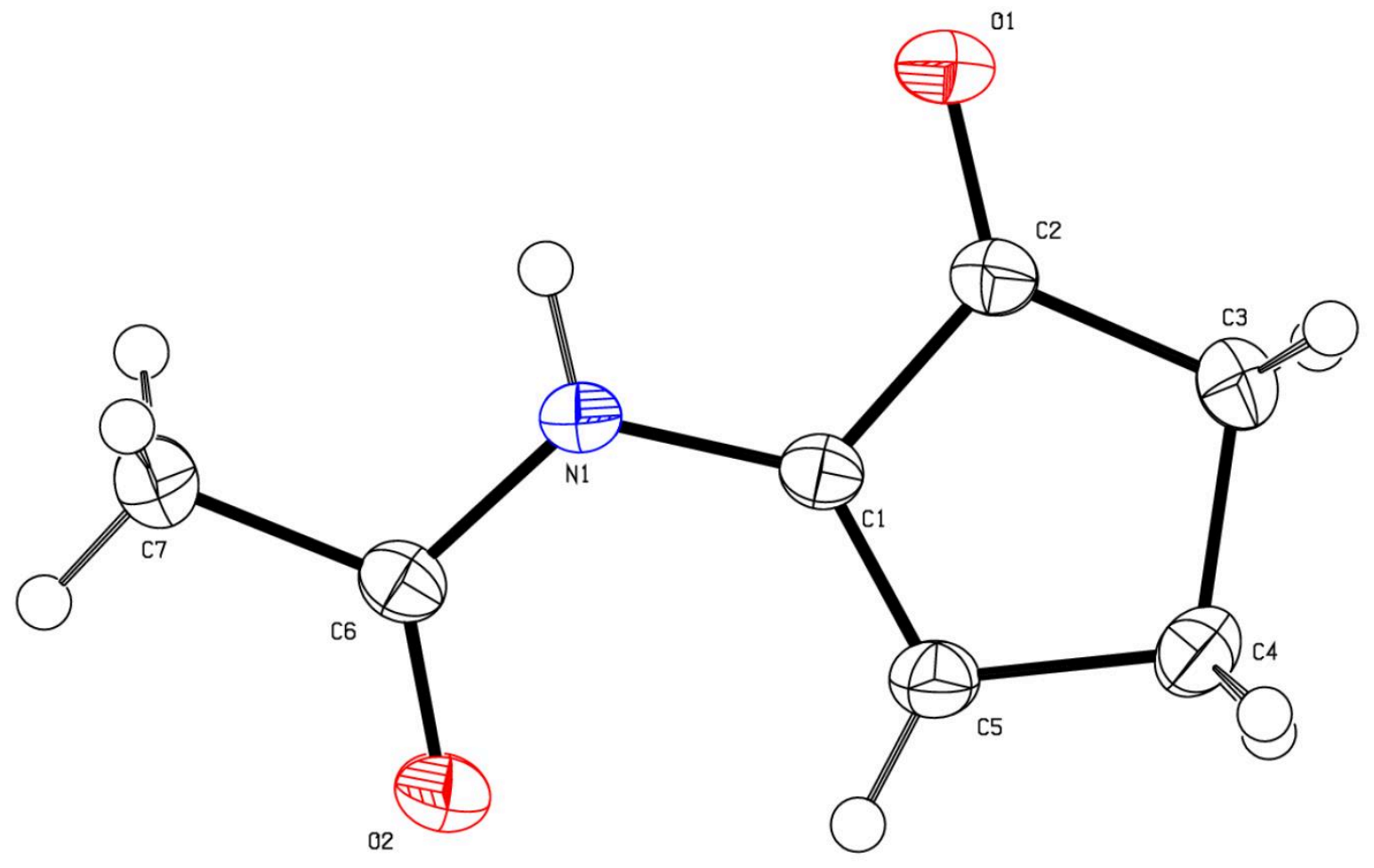

Figure S2: Structure of compound 6 (CCDC 1943033). Anisotropic displacement ellipsoids show $30 \%$ probability levels. Hydrogen atoms are drawn as circles with small radii (crystal grown from methanol). 


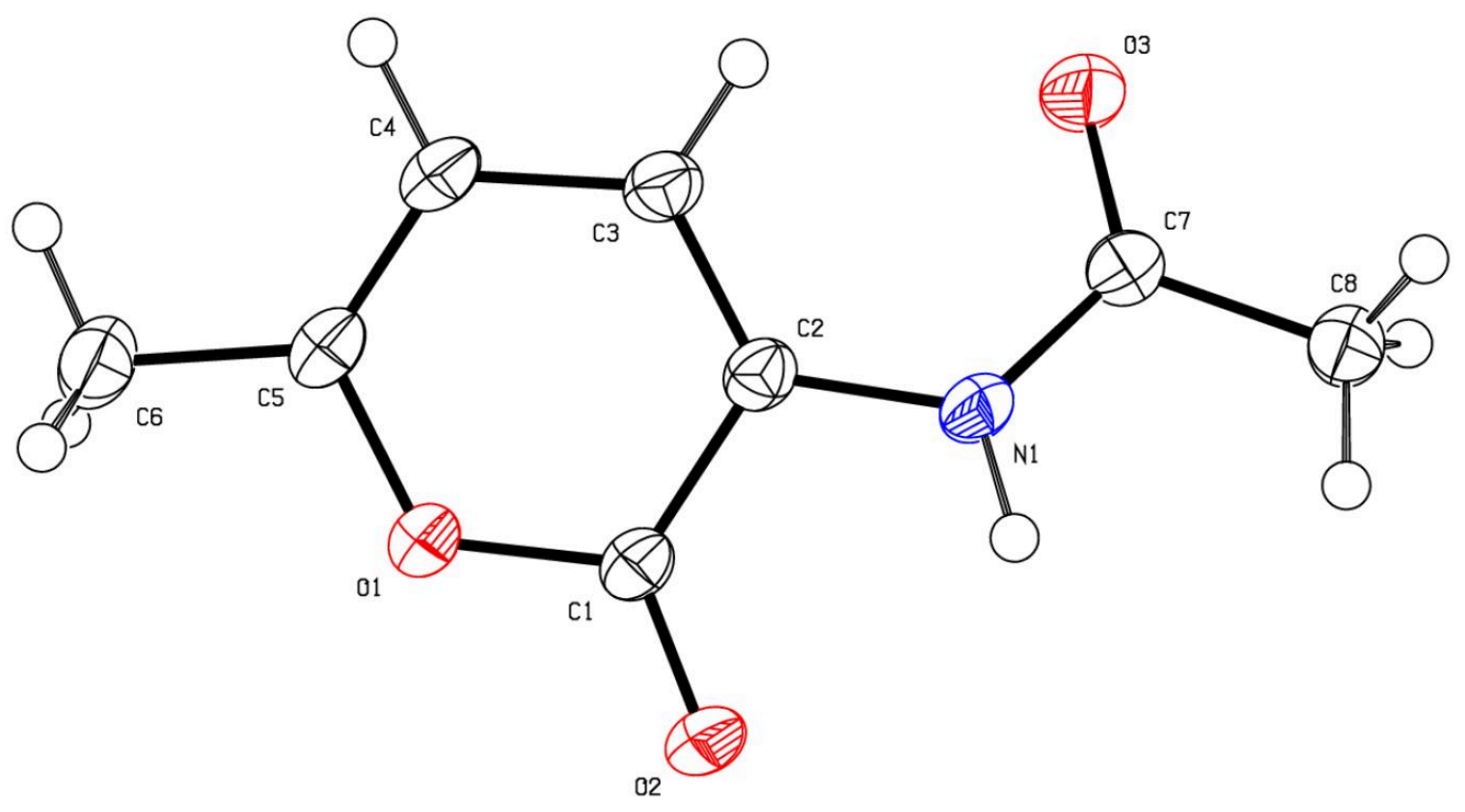

Figure S3: Structure of compound 8 (CCDC 1943034). Anisotropic displacement ellipsoids show $30 \%$ probability levels. Hydrogen atoms are drawn as circles with small radii (crystal grown from methanol). 


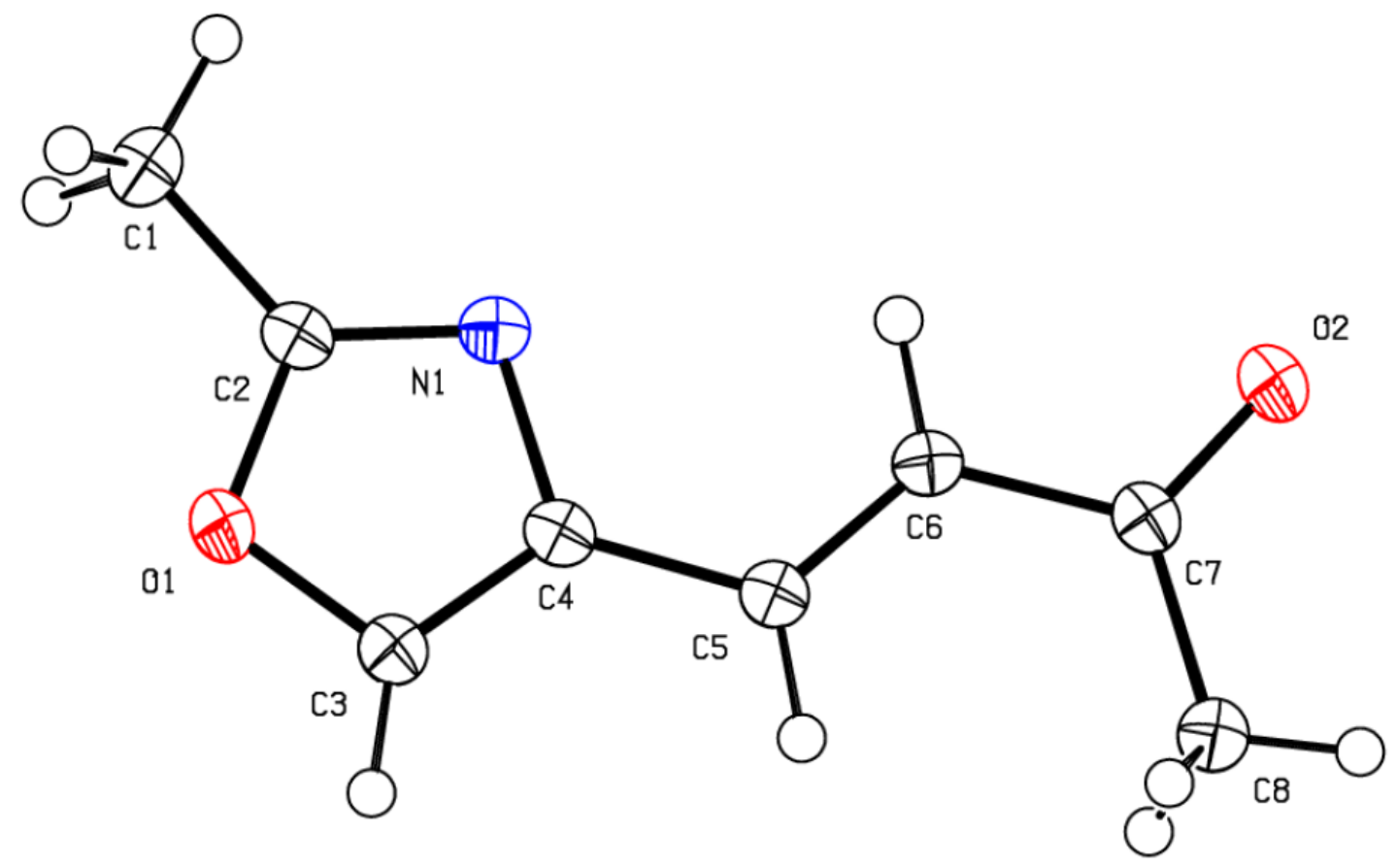

Figure S4: Structure of compound 9 (CCDC 1943035). Anisotropic displacement ellipsoids show $30 \%$ probability levels. Hydrogen atoms are drawn as circles with small radii (crystal grown from methanol). 


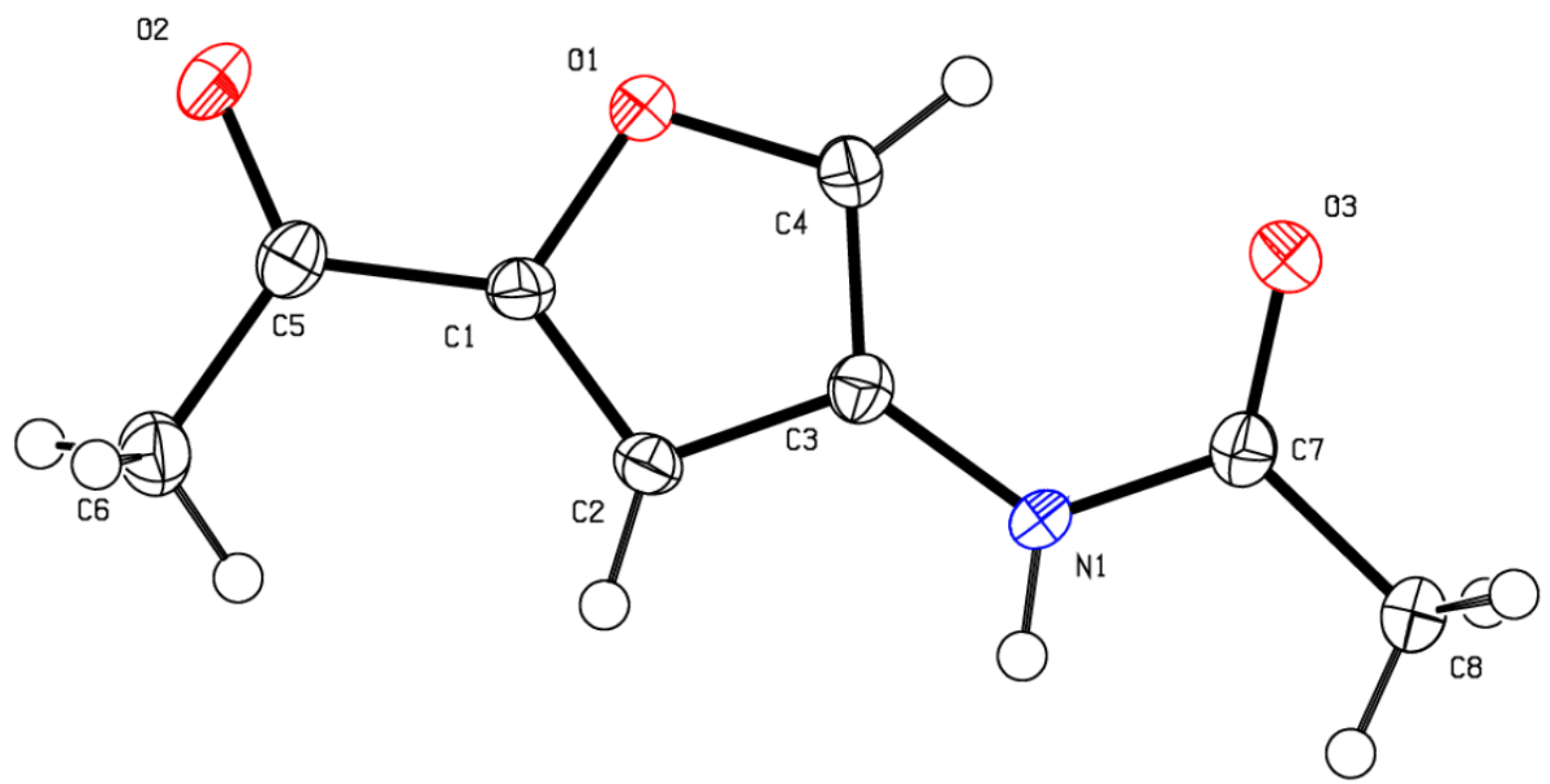

Figure S5: Structure of compound 10 (CCDC 1943036). Anisotropic displacement ellipsoids show $30 \%$ probability levels. Hydrogen atoms are drawn as circles with small radii (crystal grown from methanol). 


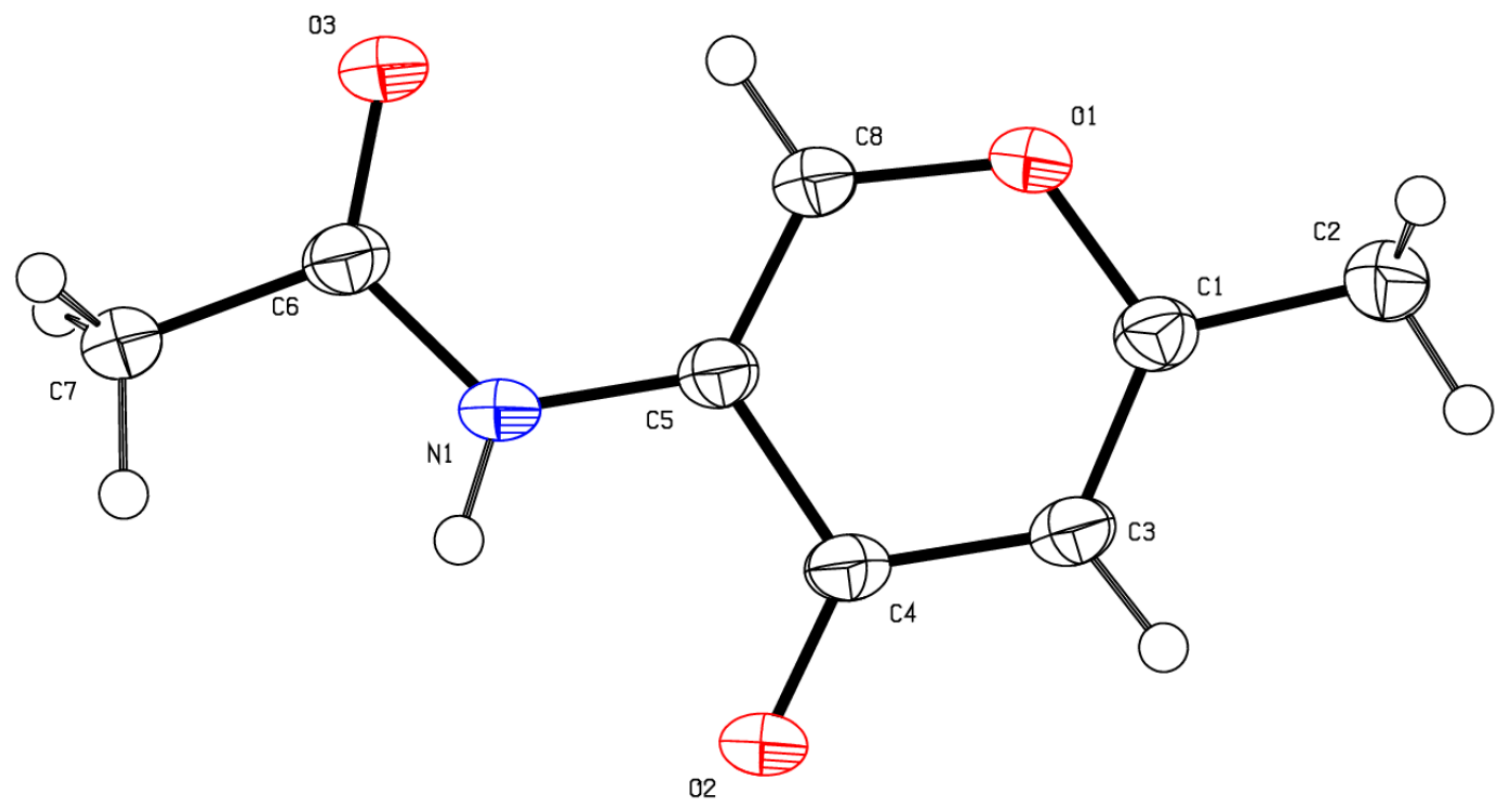

Figure S6: Structure of compound 11 (CCDC 1943037). Anisotropic displacement ellipsoids show $30 \%$ probability levels. Hydrogen atoms are drawn as circles with small radii (crystal grown from methanol). 


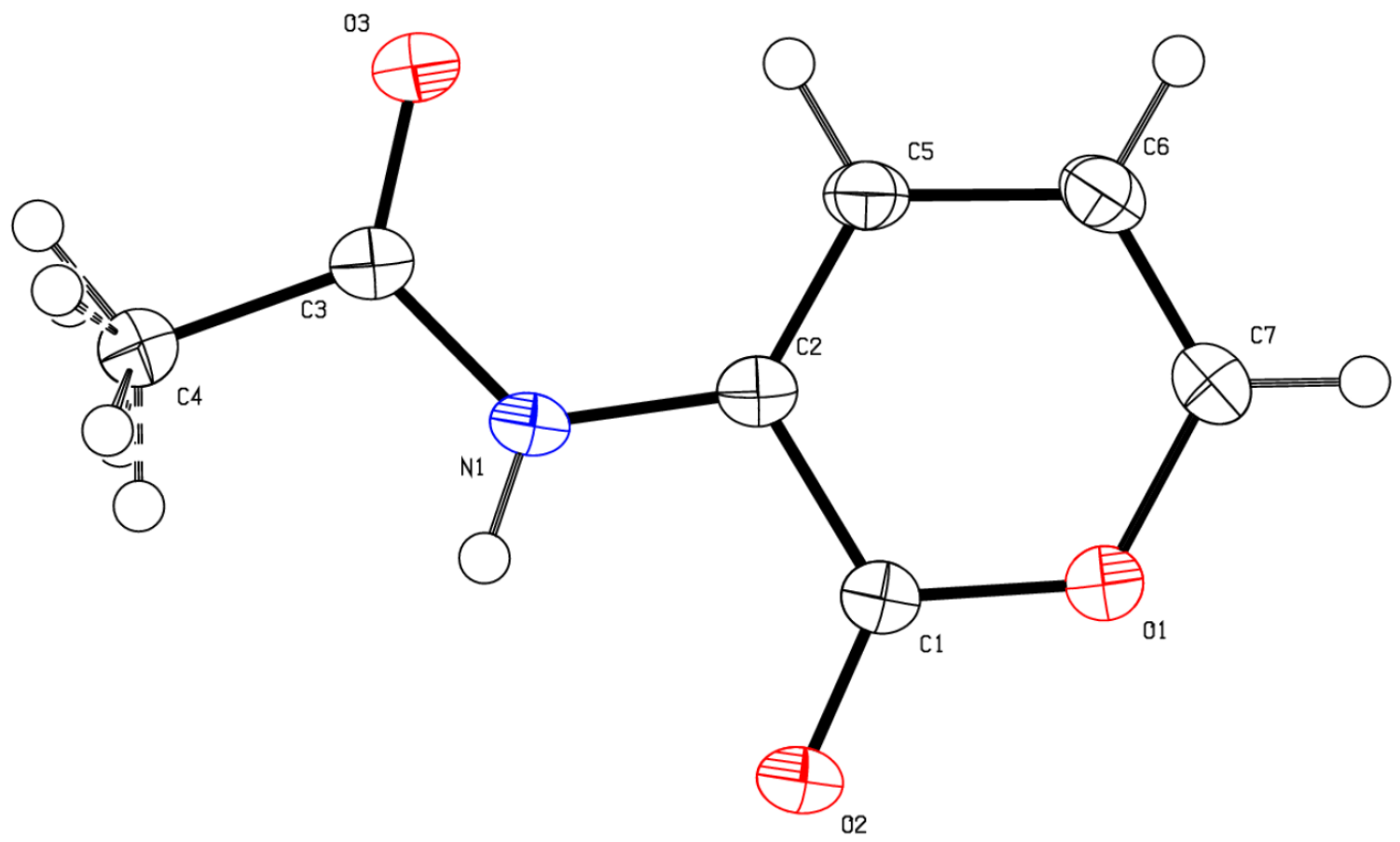

Figure S7: Structure of compound 12 (CCDC 1943038). Anisotropic displacement ellipsoids show $30 \%$ probability levels. Hydrogen atoms are drawn as circles with small radii (crystal grown from methanol). 


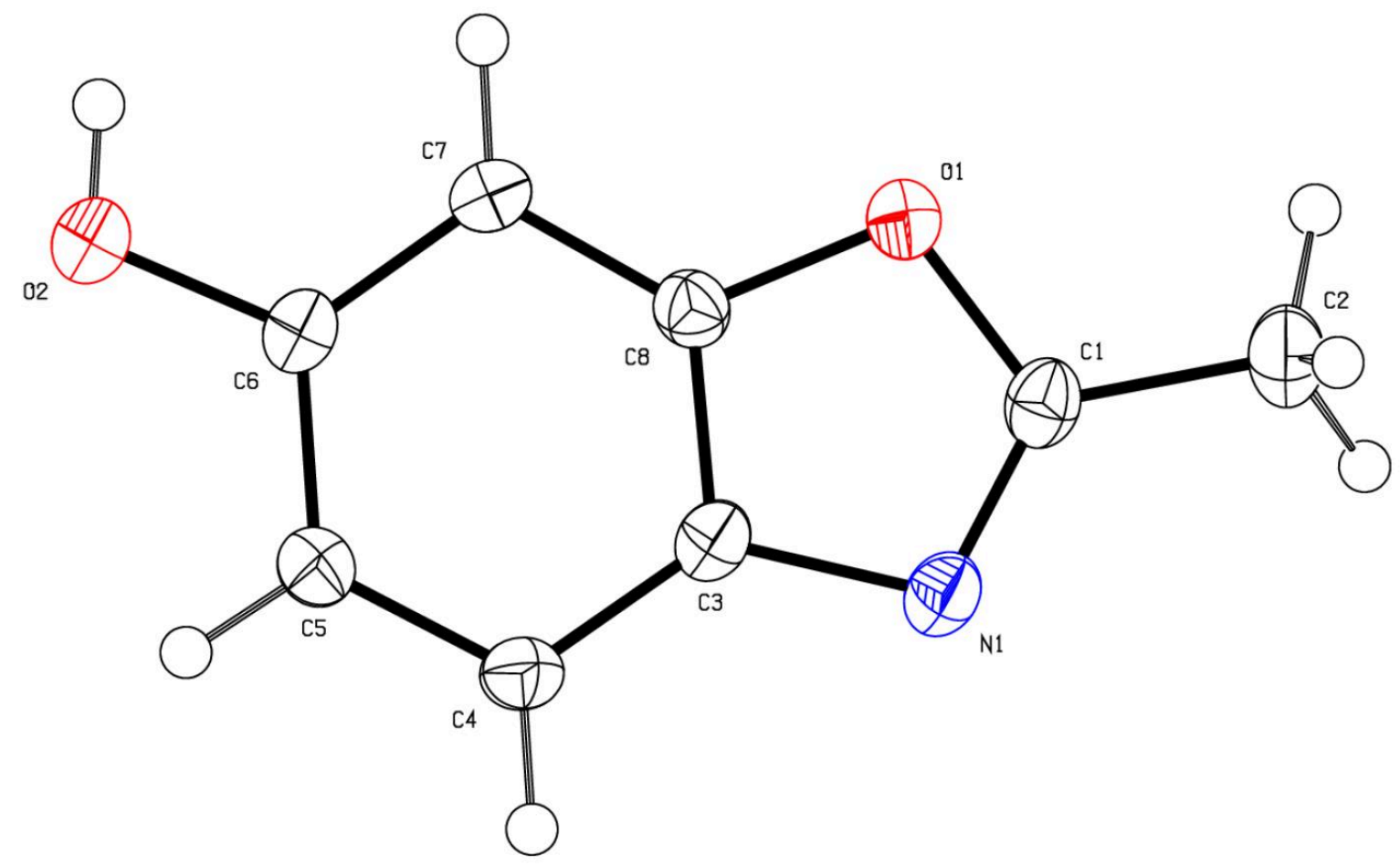

Figure S8: Structure of compound 13 (CCDC 1943039). Anisotropic displacement ellipsoids show $30 \%$ probability levels. Hydrogen atoms are drawn as circles with small radii (crystal grown from methanol). 

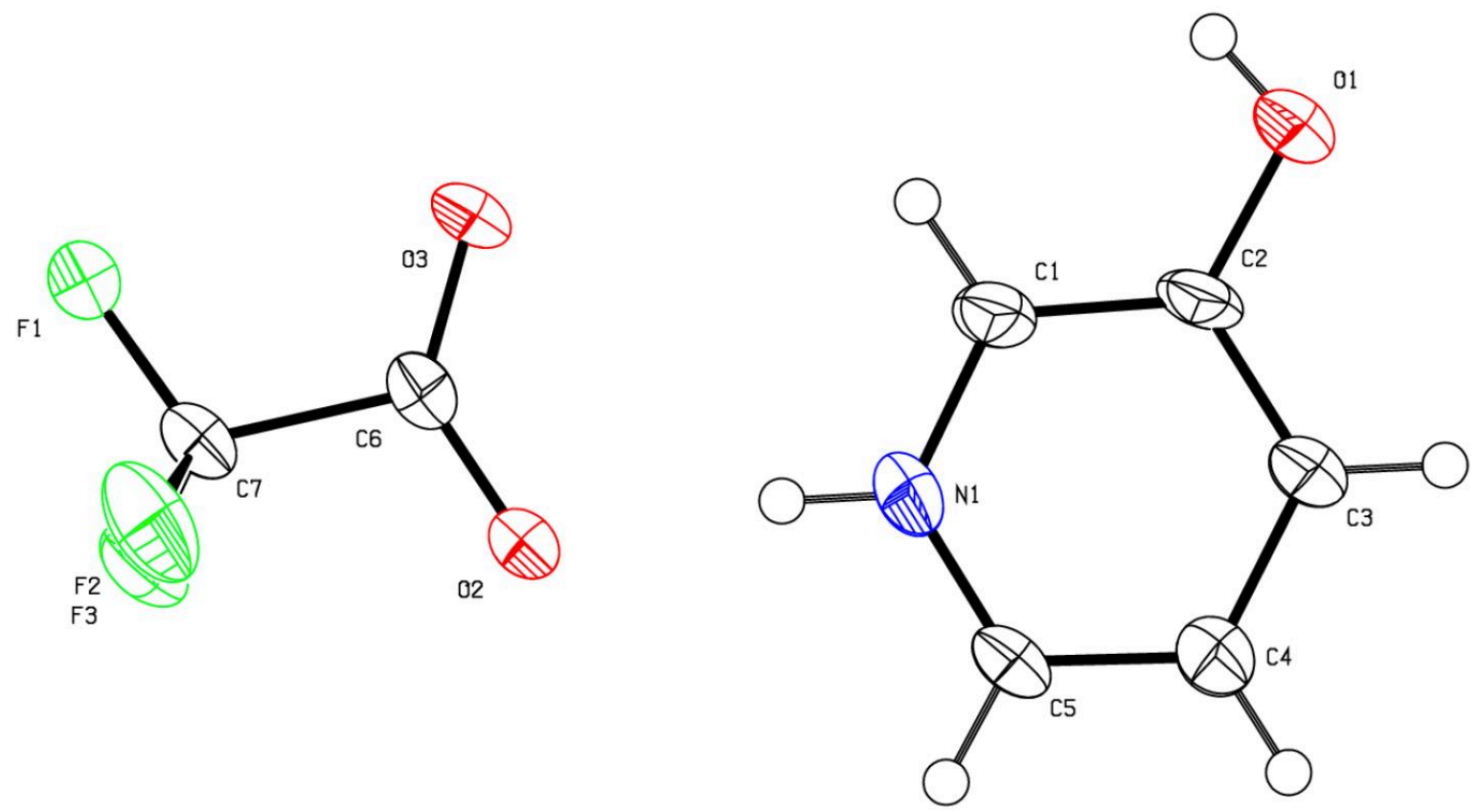

Figure S9: Structure of the trifluoroacetate salt of compound 14 (CCDC 1943040). Anisotropic displacement ellipsoids show $30 \%$ probability levels. Hydrogen atoms are drawn as circles with small radii (crystal grown from methanol). 


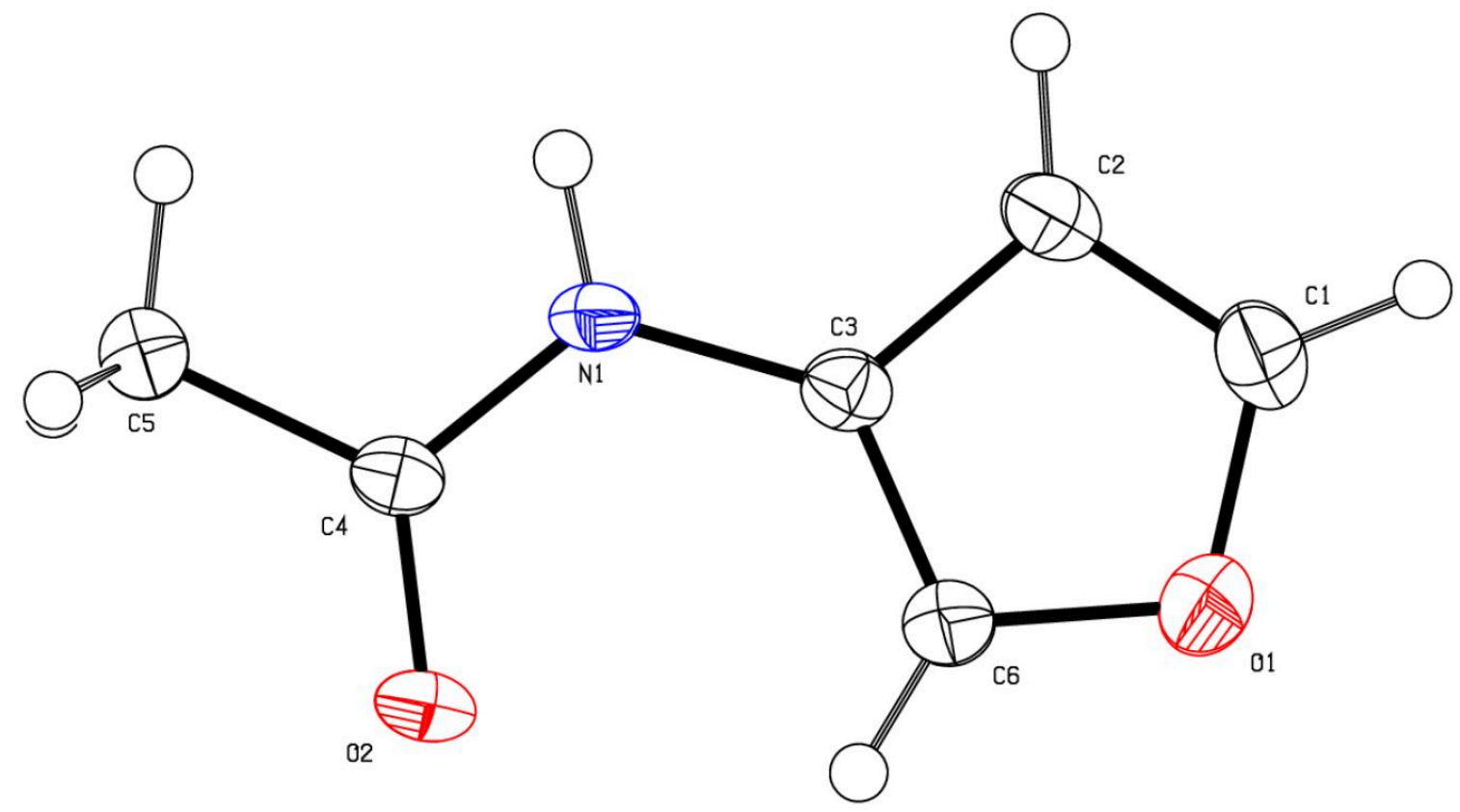

Figure S10: Structure of compound 15 (CCDC 1943041). Anisotropic displacement ellipsoids show $30 \%$ probability levels. Hydrogen atoms are drawn as circles with small radii (crystal grown from methanol). 
Scheme S1: Theoretically Determined Reaction Pathway for the Conversion $\mathbf{2 1} \rightarrow \mathbf{2 2}$ (compound numbering the same as used in main manuscript)<smiles>C[In]C1OC2OC(C)(O)NC2C(O)C1O</smiles>

21<smiles>CC1([O-])NC2OC(CO)[C@@H](O)[C@H](O)C2O1</smiles>

unstable<smiles>C[C+]#CC1OC([O-])C(NC(C)=O)[C@H](O)C1O</smiles>

INT 1<smiles>CC(=O)NC(C(C)=O)C(O)C1O[C@H]2CCCCCC1C2</smiles>

INT 2 $\begin{aligned} & \Delta G^{\ddagger}=84.3 \mathrm{~kJ} \mathrm{~mol}^{-1} \\ & \Delta G=-403.1 \mathrm{~kJ} \mathrm{~mol}^{-1}\end{aligned} \mid$ hydride shift<smiles>CC(=O)NC1C(=O)OC(C)C(O)C1O</smiles>

22<smiles>CC(=O)NC1C(=O)OC(C)C(OCO)C1O</smiles>

INT 3

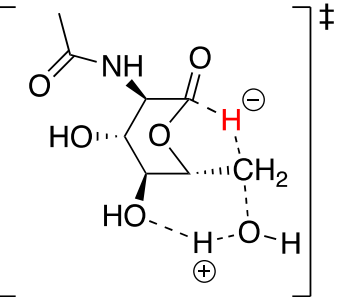

TS

Scheme S2: Theoretically Determined Reaction Pathway for the Conversion $\mathbf{2 3} \rightarrow \mathbf{2 4}$ (compound numbering the same as used in main manuscript)

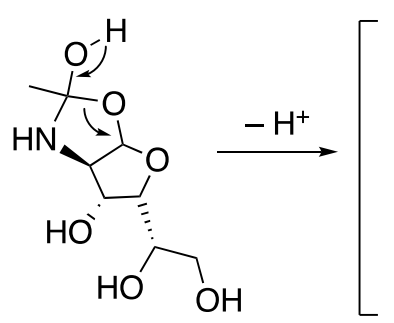

23<smiles>CC1([O-])NC2OC(C(O)CO)C(O2)C1O</smiles>

unstable

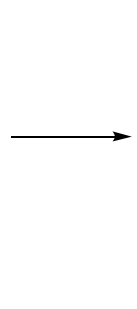

INT 1

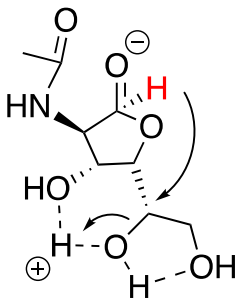

INT 2 $\begin{aligned} & \Delta G^{\ddagger}=111.1 \mathrm{~kJ} \mathrm{~mol}^{-1} \\ & \Delta G=-331.5 \mathrm{~kJ} \mathrm{~mol}^{-1}\end{aligned} \mid$ hydride shift<smiles>CC(=O)N[C@@H]1C(=O)O[C@H](CCO)[C@H]1O</smiles>

24<smiles>CC(=O)N[C@H]1C(=O)O[C@H](CO)[C@@H]1O</smiles>

INT 3

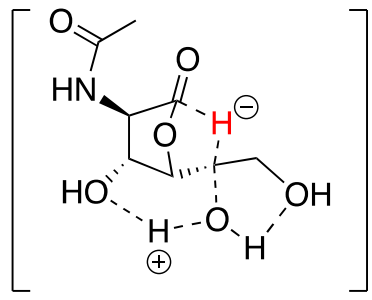

TS 


\section{Gaussian Archive Files}

Scheme S1 INT 2

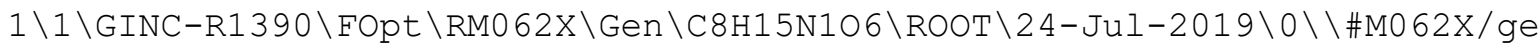
n 6D SCF=Tight INT(grid=ultrafine) OPT IOP $(2 / 17=4) \backslash \backslash 8+\mathrm{H}-\mathrm{H}$ hydride_shif $t$ TS ircf LQA opt.freq $\backslash \backslash 0,1 \backslash C,-1.9042089861,-0.4934219934,0.69994 \overline{6} 7838$ $\backslash \bar{C},-\overline{1} .361 \overline{7} 152 \overline{7} 87,0.6609115102,-0.1273451458 \backslash \mathrm{C}, 0.1051175877,0.864653276$ $6,0.0711284924 \backslash C, 0.7686387549,-0.3019673052,-0.6517795492 \backslash C,-0.0617566$ $266,-1.6778381947,-0.5377549099 \backslash \mathrm{H},-2.0679604418,-0.0969243375,1.716330$ $9777 \backslash \mathrm{H},-1.5971960797,0.5906555682,-1.1947858582 \backslash \mathrm{H}, 0.8271966853,-0.0314$ $406894,-1.7179711866 \backslash \mathrm{H},-0.8348726477,-1.6604891601,-1.3518152637 \backslash \mathrm{H}, 0.3$ $464636073,0.8185376988,1.142210846 \backslash 0,-1.9999770513,1.9412347243,0.3222$ $21542 \backslash \mathrm{H},-1.423836343,2.6518070242,-0.0764902233 \backslash \mathrm{C},-3.2655944335,-0.891$ $4156454,0.1268197549 \backslash \mathrm{H},-3.1319173391,-1.3898255978,-0.840464649 \backslash \mathrm{H},-3.7$ $952534923,-1.5600716863,0.811003313 \backslash 0,-3.9896977878,0.3474570879,-0.04$ $10688524 \backslash \mathrm{H},-2.9521466584,1.8870936858,0.0455222438 \backslash 0,-0.9677083891,-1$. $5056039258,0.7502993203 \backslash \mathrm{N}, 2.0702449506,-0.6143434746,-0.1358676578 \backslash \mathrm{H}, 2$ $.1492016903,-1.6364171522,-0.0128886555 \backslash C, 3.0450819592,0.2789558252,0$. $0359536625 \backslash 0,0.6763405265,-2.6940643653,-0.4340952685 \backslash \mathrm{C}, 4.3814200281,-$ $0.257853484,0.4948826073 \backslash \mathrm{H}, 5.1253467669,-0.0404880186,-0.2756872723 \backslash \mathrm{H}$, $4.6809835655,0.2727705414,1.4013307083 \backslash \mathrm{H}, 4.3607641048,-1.3317948728,0$. $6866852106 \backslash 0,0.3554842464,2.1538662563,-0.4608688768 \backslash \mathrm{H}, 1.3460977631,2$. $2224619743,-0.459122714 \backslash 0,2.8990162077,1.4984381972,-0.1581153732 \backslash \mathrm{H},-4$ $.8701928891,0.1662955328,-0.3946880064 \backslash \backslash$ Version=ES64L-G0 9RevE.01 \State $=1-\mathrm{A} \backslash \mathrm{HF}=-819.5264266 \backslash \mathrm{RMSD}=5.171 \mathrm{e}-09 \backslash \mathrm{RMSF}=5.111 \mathrm{e}-06 \backslash \mathrm{Dipole}=-3.1073226,2$ $.6017561,-0.21022 \backslash$ Quadrupole $=19.4645973,-14.8850109,-4.5795865,-7.7035$ $26,5.0219671,-0.4849316 \backslash \mathrm{PG}=\mathrm{C} 01 \quad[\mathrm{X}(\mathrm{C} 8 \mathrm{H} 15 \mathrm{~N} 106)] \backslash \backslash @$

Scheme S1 TS

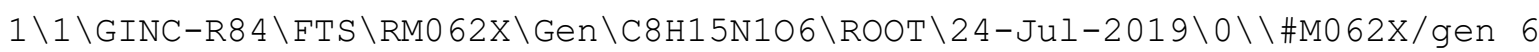
D SCF=Tight INT (grid=ultrafine) OPT=(TS, calcfC, noeigentest, maxCyC=250) IOP $(2 / 17=4) \backslash \backslash 8+\mathrm{H}-\mathrm{H}$ hydride shift TS.freq $\backslash \backslash 0,1 \backslash \mathrm{C},-1.8466302143,-0.8354$ $982237,0.5556993533 \backslash \mathrm{C},-1.62 \overline{7} 13845 \overline{9} 5,0.5062961776,-0.1529492528 \backslash \mathrm{C},-0.18$ $38463587,0.9275909277,-0.0340433601 \backslash \mathrm{C}, 0.6236472478,-0.1991723817,-0.71$ $35481433 \backslash \mathrm{C}, 0.1208195802,-1.6651434805,-0.2796924672 \backslash \mathrm{H},-2.4429656077,-0$ $.6660977708,1.4604912587 \backslash \mathrm{H},-1.804155443,0.4051864018,-1.2372797695 \backslash \mathrm{H}, 0$ $.5042065434,-0.0776438757,-1.8008530437 \backslash \mathrm{H},-0.8125111992,-1.9441024622$, $-1.0097350502 \backslash \mathrm{H}, 0.113270458,0.9907199201,1.0227635467 \backslash 0,-2.5779199703$, $1.4274708017,0.3637052527 \backslash \mathrm{H},-2.2991849172,2.3022549268,0.0372119957 \backslash \mathrm{C}$, $-2.6298948174,-1.7016151591,-0.4183515477 \backslash \mathrm{H},-2.7295871023,-1.469663003$ $1,-1.4702903089 \backslash \mathrm{H},-2.8771951333,-2.7228550761,-0.133866827 \backslash 0,-4.271224$ $4564,-0.8345699968,-0.1239344357 \backslash \mathrm{H},-3.9976712644,0.1144701621,-0.02326$ $48201 \backslash 0,-0.6845575909,-1.4598149816,0.9598631319 \backslash \mathrm{N}, 2.0283508873,-0.206$ $698932,-0.3669292788 \backslash \mathrm{H}, 2.3534704869,-1.1541330274,-0.1665520082 \backslash \mathrm{C}, 2.87$ $1814824,0.8295749136,-0.4702797635 \backslash 0,1.0043764059,-2.5415768057,-0.193$ $708627 \backslash \mathrm{C}, 4.3275263934,0.5284346106,-0.1839537615 \backslash \mathrm{H}, 4.9039380964,0.7160$ $271151,-1.0933411943 \backslash \mathrm{H}, 4.682378037,1.2228144298,0.5804841263 \backslash \mathrm{H}, 4.49674$ $62488,-0.4979608993,0.1460882917 \backslash 0,-0.1286299435,2.175874837,-0.676891$ $0835 \backslash \mathrm{H}, 0.8298112678,2.3711589583,-0.7973709929 \backslash 0,2.526449252,1.9767332$ $174,-0.774439338 \backslash \mathrm{H},-4.6419962507,-1.0926273239,0.738163117 \backslash \backslash$ Version $=\mathrm{ES}$ 64L-G0 9RevE.01 \State $=1-A \backslash H F=-819.4858719 \backslash \mathrm{RMSD}=3.267 \mathrm{e}-09 \backslash \mathrm{RMSF}=6.192 \mathrm{e}-06$ $\backslash$ Dipole $=-3.1648307,-0.1105508,0.1960875 \backslash$ Quadrupole $=20.1128266,-15.5905$ $556,-4.5222711,5.6736618,-2.7249168,2.1287598 \backslash P G=C 01 \quad[X(C 8 H 15 N 106)] \backslash \backslash @$

Scheme S1 INT 3

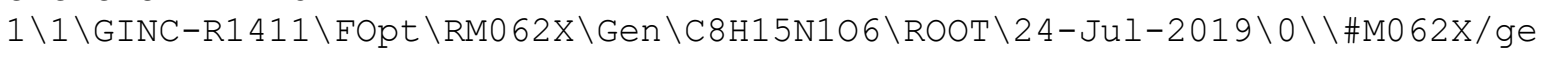


n 6D SCF=Tight INT(grid=ultrafine) OPT IOP(2/17=4) \/8+H-H_hydride_shif

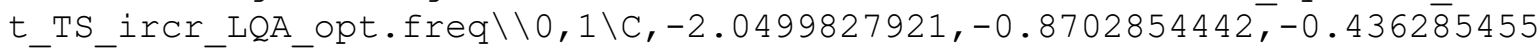
$9 \bar{\top} \mathrm{C}, \overline{-}-1.45 \overline{2} 552 \overline{6} 555,0.3960004187,-1.0170515637 \backslash \mathrm{C},-0.1070783565,0.7084918$ $565,-0.3812183396 \backslash \mathrm{C}, 0.8372257937,-0.4682600206,-0.6506189837 \backslash \mathrm{C}, 0.17120$ $56622,-1.8513773947,-0.4873377813 \backslash \mathrm{H},-2.1550602496,-0.7560694773,0.6484$ $442861 \backslash \mathrm{H},-1.3001802108,0.2523996987,-2.0998121629 \backslash \mathrm{H}, 1.1161466052,-0.40$ $10719319,-1.7152545293 \backslash \mathrm{H},-3.2532724082,-1.3707356417,-2.1482609777 \backslash \mathrm{H},-$ $0.2427922182,0.8363244185,0.7042627843 \backslash 0,-2.3377263704,1.4775496118,-0$ $.7942079574 \backslash \mathrm{H},-1.8060170265,2.2697449385,-0.994769004 \backslash \mathrm{C},-3.372008577,-$ $1.2454272874,-1.0679132835 \backslash \mathrm{H},-4.0993896775,-0.4520210656,-0.8822700143$ $\backslash \mathrm{H},-3.7430579255,-2.1806485092,-0.6432334124 \backslash \mathrm{O},-2.2740650247,1.1352210$ $894,2.0073112426 \backslash \mathrm{H},-2.5066356681,1.4590282458,1.1185367842 \backslash 0,-1.143830$ $5778,-1.9712082907,-0.6839009105 \backslash \mathrm{N}, 2.0316463958,-0.5291876332,0.177248$ $1947 \backslash \mathrm{H}, 2.308390071,-1.4755658209,0.4128278464 \backslash \mathrm{C}, 2.8766047204,0.4890193$ $352,0.4505732467 \backslash 0,0.8462166546,-2.8262522737,-0.2740503959 \backslash \mathrm{C}, 4.119680$ $5309,0.1114086119,1.2265343773 \backslash \mathrm{H}, 4.9907787356,0.3061428167,0.596080740$ $5 \backslash \mathrm{H}, 4.1907513237,0.7633380227,2.0992582277 \backslash \mathrm{H}, 4.1328427507,-0.931367341$ $9,1.548348326 \backslash 0,0.2961323672,1.9013349636,-1.0037847218 \backslash \mathrm{H}, 1.1888076776$ , $2.1115653039,-0.6521462113 \backslash 0,2.684396618,1.6560709664,0.1168541247 \backslash \mathrm{H}$, $-2.9553621684,1.4790748348,2.5986675238 \backslash \backslash V e r s i o n=E S 64 L-G 09 R e v E .01 \backslash$ Stat $e=1-A \backslash H F=-819.6590869 \backslash \mathrm{RMSD}=4.172 \mathrm{e}-09 \backslash \mathrm{RMSF}=1.217 \mathrm{e}-06 \backslash \mathrm{Dipole}=-0.1530084$, $0.2406513,0.2277479 \backslash$ Quadrupole $=9.656184,-10.3943714,0.7381873,-5.10163$ $21,0.5904959,1.2743099 \backslash \mathrm{PG}=\mathrm{C} 01 \quad[\mathrm{X}(\mathrm{C} 8 \mathrm{H} 15 \mathrm{~N} 106)] \backslash \backslash @$

\section{Scheme S2 INT 2}

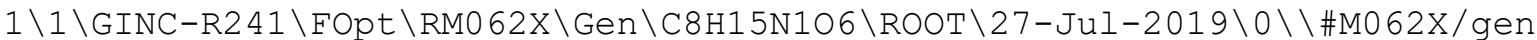

6D SCF=Tight INT (grid=ultrafine) OPT IOP $(2 / 17=4) \backslash \backslash 4-\mathrm{H}+\mathrm{H}$ hydride ring

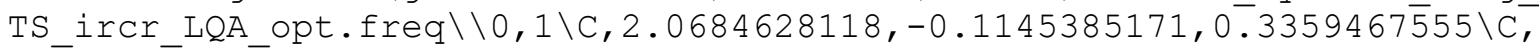
$1 . \overline{4} 1674 \overline{8} 186 \overline{4},-0.1236689361,-1.0630682665 \backslash \mathrm{C}, 0.1313972825,0.7302847991,-$ $1.0373275079 \backslash \mathrm{C},-0.9840351516,-0.1077676734,-0.4140118952 \backslash \mathrm{C},-1.40548339$ $41,-1.1329991776,-1.4735341619 \backslash \mathrm{H}, 1.3957626349,-0.574632499,1.073424393$ $\backslash \mathrm{H}, 2.1479020824,0.3921146371,-1.734356105 \backslash \mathrm{H},-0.618915619,-0.6124612151$ , $0.4802852212 \backslash \mathrm{H},-1.2128355786,-2.1914483871,-1.2644962044 \backslash \mathrm{H},-0.1642569$ $483,1.0538944639,-2.0371177021 \backslash 0,1.02594361,-1.3478583242,-1.425326115$ $8 \backslash \mathrm{C}, 3.3805548746,-0.8515723743,0.3623002011 \backslash \mathrm{H}, 3.1882038692,-1.90752336$ $88,0.149121305 \backslash \mathrm{H}, 4.0450118554,-0.4383380538,-0.4090485184 \backslash 0,3.90858688$ $38,-0.6313423931,1.6674526726 \backslash \mathrm{H}, 4.8037514718,-0.9913760848,1.711540935$ $4 \backslash 0,2.3153393633,1.2615017498,0.7369049468 \backslash \mathrm{N},-2.0858398989,0.795863731$ $4,-0.0652381147 \backslash \mathrm{C},-2.9194324848,0.6228838368,1.0464312647 \backslash 0,-2.0023128$ $854,-0.7383929206,-2.449697109 \backslash \mathrm{C},-2.3316813811,-0.1110631858,2.2340013$ $92 \backslash \mathrm{H},-2.9928772714,0.0498524818,3.0842689797 \backslash \mathrm{H},-2.2724323333,-1.184671$ $428,2.0287179531 \backslash \mathrm{H},-1.3236338249,0.2390703717,2.4763393254 \backslash 0,0.2428372$ $067,2.0034896934,-0.2398573451 \backslash \mathrm{H},-0.6372666808,2.0906987263,0.21323052$ $97 \backslash \mathrm{H}, 2.8521967823,1.2446001316,1.5492939898 \backslash 0,-4.0341071197,1.09054755$ $58,1.0537636364 \backslash \mathrm{H},-2.6312312612,1.0660023285,-0.8858070058 \backslash \mathrm{H}, 1.0895389$ $18,1.8807450317,0.3959705504 \backslash \backslash$ Version=ES 64L-G09RevE.01 $\backslash$ State $=1-A \backslash H F=-8$ $19.5112931 \backslash \mathrm{RMSD}=5.983 e-09 \backslash \mathrm{RMSF}=1.750 e-05 \backslash \mathrm{Dipole}=1.7848439,1.0959516,2$. $3455131 \backslash$ Quadrupole=1.2404542,2.4269801,-3.6674343,2.5554842,3.001461, $5.5398472 \backslash \mathrm{PG}=\mathrm{C} 01 \quad[\mathrm{X}(\mathrm{C} 8 \mathrm{H} 15 \mathrm{~N} 106)] \backslash \backslash @$

Scheme S2 TS

$1 \backslash 1 \backslash G I N C-R 272 \backslash F T S \backslash R M 062 X \backslash G e n \backslash C 8 H 15 N 106 \backslash R 00 T \backslash 25-J u l-2019 \backslash 0 \backslash \backslash \# M 062 X / g e n$ 6D SCF=Tight INT (grid=ultrafine) OPT=(TS, calcfc, noeigentest, maxcyc=250 ) $\operatorname{IOP}(2 / 17=4) \backslash \backslash 4-\mathrm{H}+\mathrm{H}$ hydride ring TS.freq $\backslash \backslash 0,1 \backslash \mathrm{C}, 1.9222683013,-0.26070$ $88046,0.3281390676 \backslash \mathrm{C}^{-}{ }^{-1} .17697 \overline{6} 7552,0.0751074488,-0.9649960059 \backslash \mathrm{C}, 0.26358$ $01696,1.2803343265,-0.6965881586 \backslash \mathrm{C},-0.9415886557,0.611425904,-0.019624$ $6718 \backslash \mathrm{C},-0.6025879037,-0.9339367375,-0.071218647 \backslash \mathrm{H}, 1.693188746,0.218904$ $095,1.2736694876 \backslash \mathrm{H}, 1.9140735713,0.24501961,-1.7552595158 \backslash \mathrm{H},-1.02012706$ 
$85,0.9345726544,1.0236544923 \backslash \mathrm{H}, 0.2792612111,-1.0218602293,0.75211663 \backslash \mathrm{H}$ $,-0.0343621981,1.7316357509,-1.6501456685 \backslash 0,0.3325071503,-0.9666806628$ $,-1.2925676699 \backslash \mathrm{C}, 2.7360652558,-1.5205315058,0.3985331561 \backslash \mathrm{H}, 2.085065210$ $7,-2.3572123466,0.6694170501 \backslash \mathrm{H}, 3.1692684616,-1.7209702428,-0.589507313$ $8 \backslash 0,3.7408022797,-1.2815827107,1.3763315559 \backslash \mathrm{H}, 4.2662387121,-2.08435005$ $45,1.4949042484 \backslash 0,3.3308315119,0.9012864029,0.0443027277 \backslash \mathrm{N},-2.17295339$ $77,0.9221766162,-0.711962746 \backslash C,-3.4173300189,0.5211715583,-0.243991546$ $5 \backslash 0,-1.5010088965,-1.7815445696,-0.0246989823 \backslash C,-3.5371735732,0.282369$ $8473,1.2451861367 \backslash \mathrm{H},-4.5917854714,0.1408197812,1.4783032344 \backslash \mathrm{H},-2.97215$ $59442,-0.619277353,1.4977856044 \backslash \mathrm{H},-3.145257589,1.1274410483,1.82091388$ $95 \backslash 0,0.9849027893,2.233824814,0.0895329508 \backslash \mathrm{H}, 0.408883765,2.9907654317$, $0.2662813789 \backslash \mathrm{H}, 3.920371914,0.6900580232,0.7947992459 \backslash 0,-4.3598677976,0$ $.4344736342,-1.0041798935 \backslash \mathrm{H},-2.1294403144,0.7883540657,-1.7187271249 \backslash \mathrm{H}$ $, 2.8580352342,1.7497952847,0.1997455182 \backslash \backslash$ Version=ES64L-G0 9RevE.01 \Stat $\mathrm{e}=1-\mathrm{A} \backslash \mathrm{HF}=-819.4734243 \backslash \mathrm{RMSD}=4.941 \mathrm{e}-09 \backslash \mathrm{RMSF}=1.185 \mathrm{e}-05 \backslash \mathrm{Dipole}=4.8721711,1$ $.7585014,1.5368703 \backslash$ Quadrupole $=-3.2955827,3.6376303,-0.3420476,-7.30952$ $43,-1.4588699,-1.6480613 \backslash \mathrm{PG}=\mathrm{C} 01 \quad[\mathrm{X}(\mathrm{C} 8 \mathrm{H} 15 \mathrm{~N} 1 \mathrm{O} 6)] \backslash \backslash \mathrm{Q}$

Scheme S2 INT 3

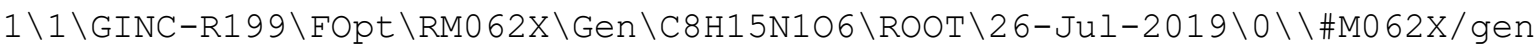
6D SCF=Tight INT (grid=ultrafine) OPT IOP $(2 / 17=4) \backslash \backslash 4-\mathrm{H}+\mathrm{H}$ _hydride_ring_ TS_ircf_LQA_opt.freq $\backslash \backslash 0,1 \backslash C, 2.0195764587,-0.6111677601,0.7915248 \overline{4} 25 \backslash \mathrm{C}$, $1 . \overline{3} 1465 \overline{7} 237 \overline{4},-0.0673200438,-0.4455587921 \backslash \mathrm{C}, 0.2897206064,1.0419987217,-$ $0.1528247365 \backslash C,-0.9807097913,0.2433305571,0.1053941289 \backslash C,-0.7790613783$ $,-0.9833389597,-0.7914725307 \backslash \mathrm{H}, 2.3479310916,0.2193924445,1.4234164066 \backslash$ $\mathrm{H}, 2.0470408875,0.2620376206,-1.1808726396 \backslash \mathrm{H},-0.9490768812,-0.101191787$ $9,1.1468083684 \backslash \mathrm{H}, 1.3229271024,-1.2183466775,1.3812435352 \backslash \mathrm{H}, 0.167723401$ $5,1.6535353043,-1.0593624828 \backslash 0,0.5357867005,-1.1184129086,-1.063068508$ $9 \backslash \mathrm{C}, 3.2357153642,-1.4351331714,0.4052677487 \backslash \mathrm{H}, 3.6457965992,-1.94524247$ $4,1.2861394039 \backslash \mathrm{H}, 2.9541470291,-2.1937658452,-0.3370449279 \backslash 0,4.18442676$ $76,-0.5226189362,-0.1325199956 \backslash \mathrm{H}, 4.8975570405,-1.0123198431,-0.5625264$ $584 \backslash 0,3.1897017343,2.2305315795,-0.4925129591 \backslash N,-2.195803286,0.9781443$ $845,-0.1183233007 \backslash C,-3.4589981358,0.5371969254,0.2585563569 \backslash 0,-1.62496$ $45189,-1.723141303,-1.2032562651 \backslash C,-3.5099727246,-0.5309369545,1.32844$ $57632 \backslash \mathrm{H},-4.5550859764,-0.6927787654,1.5877468376 \backslash \mathrm{H},-3.0795039116,-1.46$ $12124801,0.9458337013 \backslash \mathrm{H},-2.95585177,-0.228031181,2.2225172093 \backslash 0,0.6938$ $052959,1.8380880357,0.9363516552 \backslash \mathrm{H}, 0.0113498586,2.5118170084,1.0778649$ $763 \backslash \mathrm{H}, 3.73521889,1.4568386684,-0.2751912098 \backslash 0,-4.4481101498,1.03011675$ $91,-0.2371504563 \backslash \mathrm{H},-2.236833912,1.5237802099,-0.9742447582 \backslash \mathrm{H}, 2.5933003$ $707,2.3391128725,0.2637640878 \backslash \backslash$ Version=ES64L-G0 9RevE.01 \State $=1-A \backslash H F=-$ 819.6378236 $\backslash \mathrm{RMSD}=7.071 \mathrm{e}-09 \backslash \mathrm{RMSF}=1.240 \mathrm{e}-05 \backslash \mathrm{Dipole}=1.6302967,-0.4794367$, $1.3337724 \backslash$ Quadrupole $=-6.3520974,1.5993433,4.7527541,-9.7941287,-3.8913$ $203,-1.3450761 \backslash P G=C 01 \quad[X(C 8 H 15 N 106)] \backslash \backslash @$ 
Table S1: Raw Data.

Unless otherwise noted, units are in Hartree.

\begin{tabular}{|c|c|c|c|c|c|c|c|c|}
\hline Species & ZPVE & TC & $\begin{array}{c}\mathrm{S}(\mathbf{J} \\
\mathrm{mol}^{-1} \\
\left.\mathbf{K}^{-1}\right)\end{array}$ & $\begin{array}{c}\operatorname{CCSD}(T) / 6- \\
31 G(d)\end{array}$ & $\begin{array}{l}\text { MP2/6- } \\
\text { 31G(d) }\end{array}$ & MP2/G3MP2large & HLC & G(MP2,CC) \\
\hline $\begin{array}{l}\text { Scheme } \\
\text { S1 INT } \\
\quad 2\end{array}$ & 0.239225 & 0.061381 & 777.55 & -817.606525 & $\begin{array}{l}- \\
817.450056\end{array}$ & -818.426965 & $\begin{array}{l}- \\
0.414172\end{array}$ & -818.881547 \\
\hline $\begin{array}{l}\text { Scheme } \\
\text { S1 TS }\end{array}$ & 0.236379 & 0.060881 & 776.55 & -817.569887 & $\begin{array}{l}- \\
817.407454\end{array}$ & -818.385769 & $\begin{array}{l}- \\
0.414172\end{array}$ & -818.849424 \\
\hline $\begin{array}{c}\text { Scheme } \\
\text { S1 INT } \\
\mathbf{3}\end{array}$ & 0.237948 & 0.065152 & 855.95 & -817.74739 & $\begin{array}{l}- \\
817.589577\end{array}$ & -818.563092 & $\begin{array}{l}- \\
0.414172\end{array}$ & -819.035090 \\
\hline $\begin{array}{c}\text { Scheme } \\
\text { S2 INT } \\
2\end{array}$ & 0.236089 & 0.063245 & 818.54 & -817.598823 & $\begin{array}{l}- \\
817.440759\end{array}$ & -818.416634 & $\begin{array}{l}- \\
0.414172\end{array}$ & -818.883812 \\
\hline $\begin{array}{l}\text { Scheme } \\
\text { S2 TS }\end{array}$ & 0.235782 & 0.062162 & 786.48 & -817.558314 & $\begin{array}{l}- \\
817.396357\end{array}$ & -818.376626 & $\begin{array}{l}- \\
0.414172\end{array}$ & -818.841479 \\
\hline $\begin{array}{c}\text { Scheme } \\
\text { S2 INT } \\
\mathbf{3}\end{array}$ & 0.238514 & 0.064964 & 833.90 & -817.726639 & $\begin{array}{l}- \\
817.568502\end{array}$ & -818.543330 & $\begin{array}{l}- \\
0.414172\end{array}$ & -819.010083 \\
\hline
\end{tabular}


$400 \mathrm{MHz}{ }^{1} \mathrm{H}$ NMR spectrum of a 3:1 mixture of compounds 4 and 5 recorded in $\mathrm{CD}_{3} \mathrm{OD}$

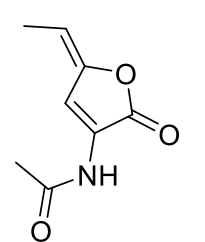

4

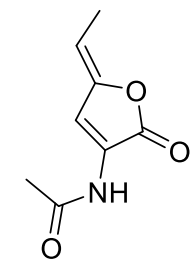

5

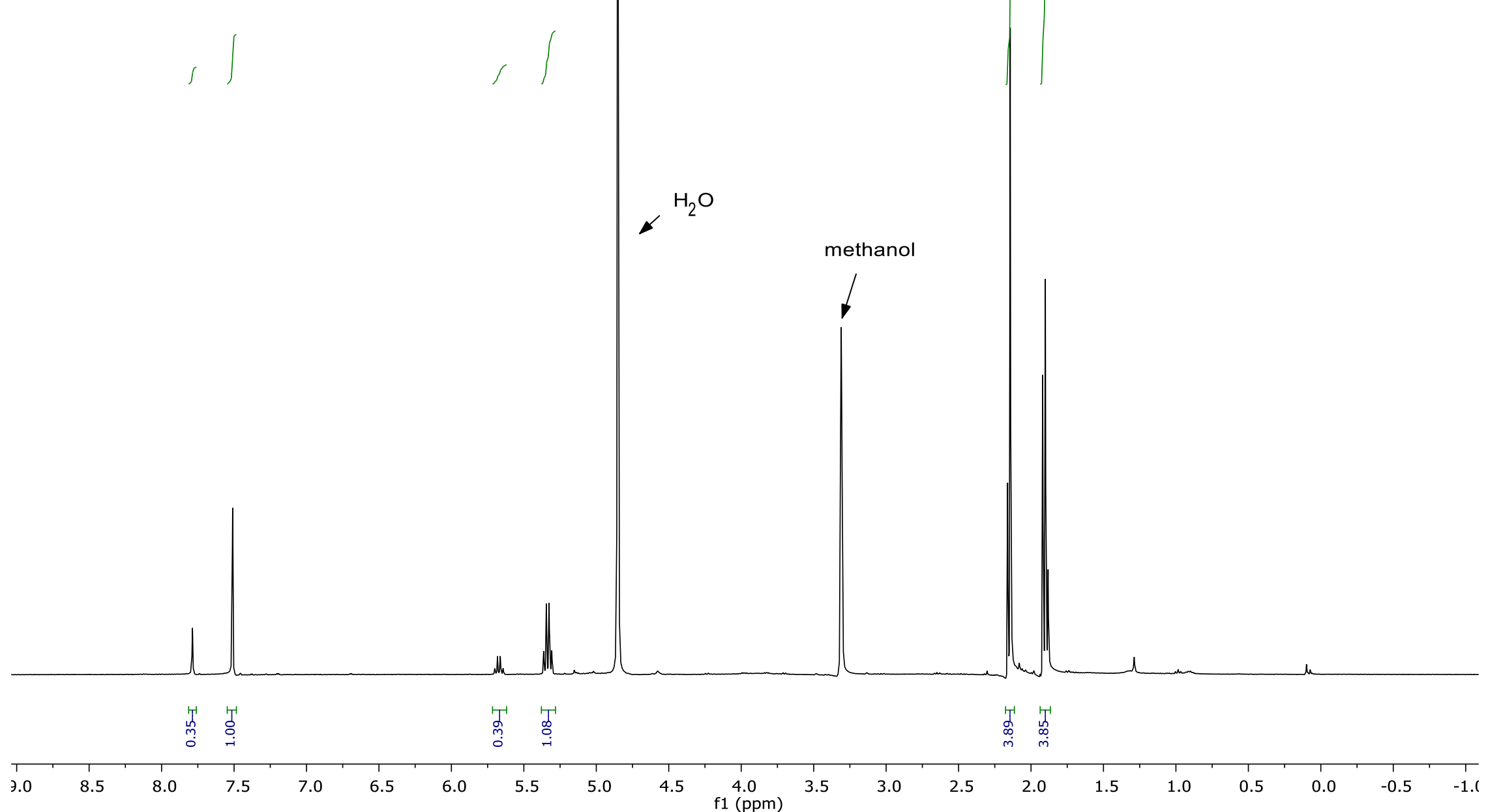


$100 \mathrm{MHz}{ }^{13} \mathrm{C}\left\{{ }^{1} \mathrm{H}\right\}$ NMR spectrum of a 3:1 mixture of compounds 4 and $\mathbf{5}$ recorded in $\mathrm{CDCl}_{3}$

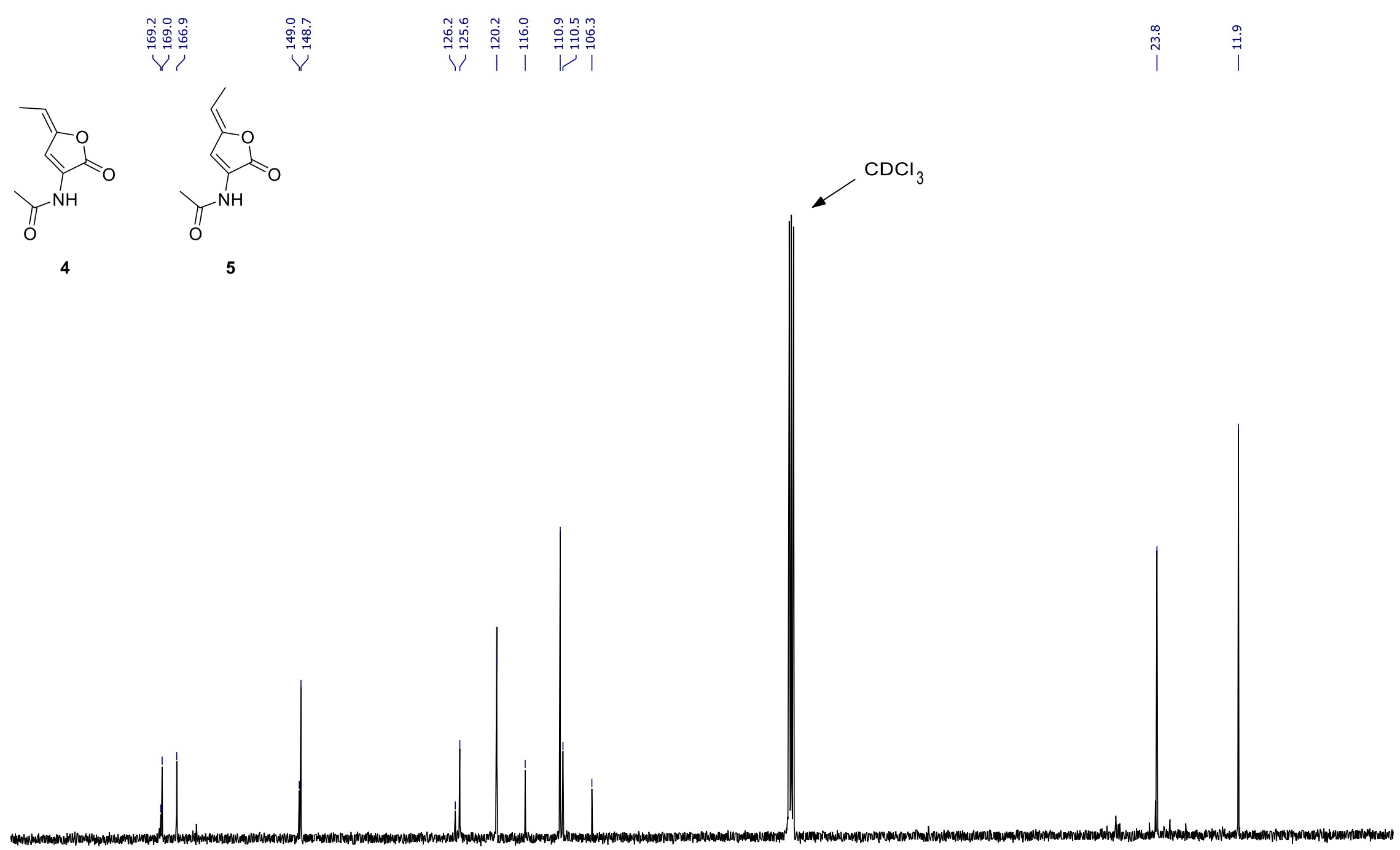

$T$

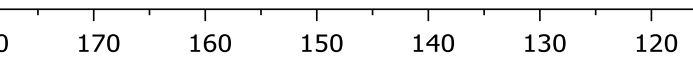

110

$100 \quad 90$

f1 $\stackrel{90}{(\mathrm{ppm})}$ 


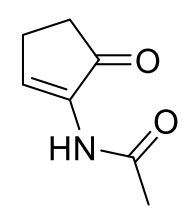

6

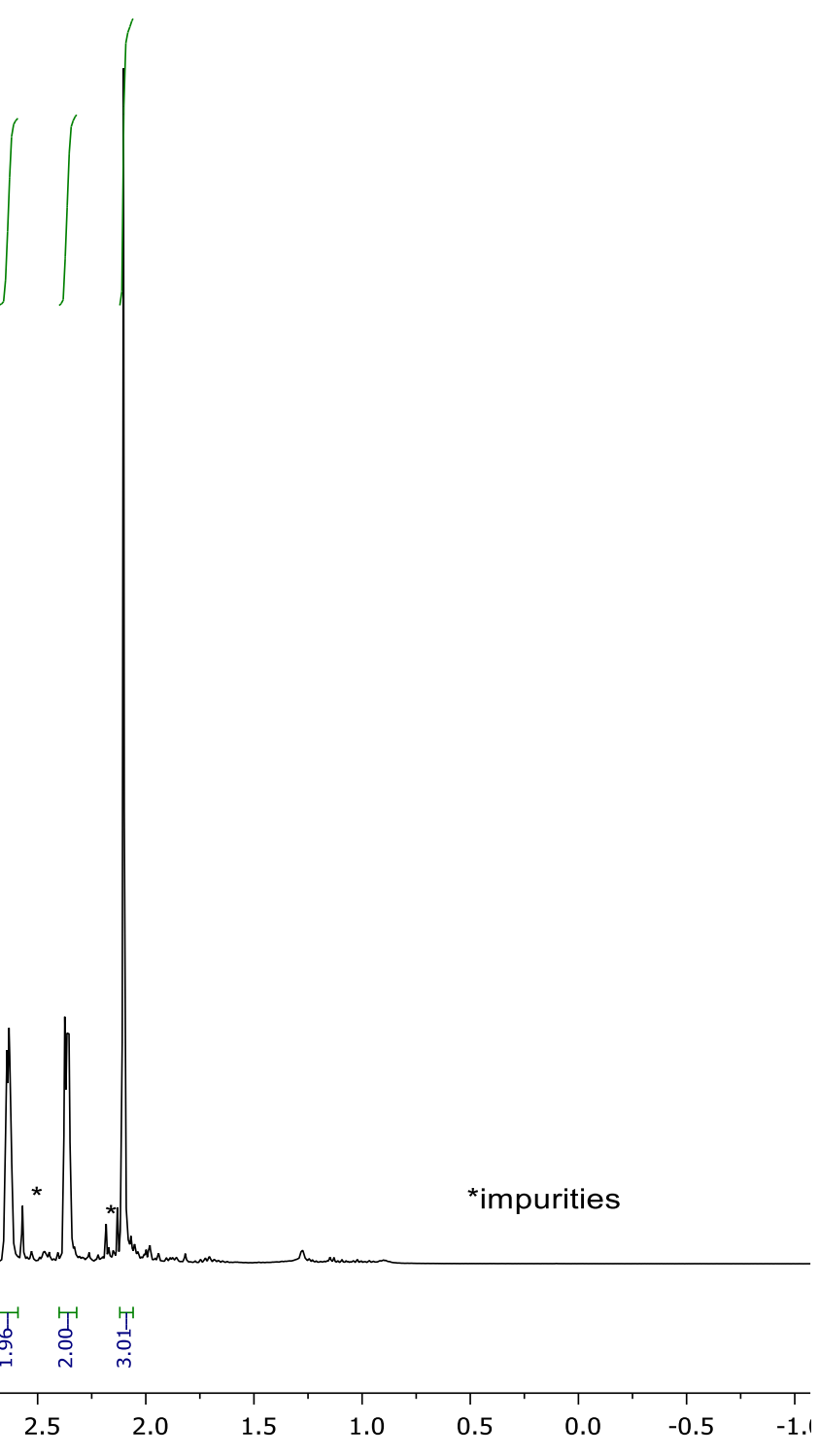


$100 \mathrm{MHz}{ }^{13} \mathrm{C}\left\{{ }^{1} \mathrm{H}\right\}$ NMR spectrum of compound 6 recorded in $\mathrm{CD}_{3} \mathrm{OD}$

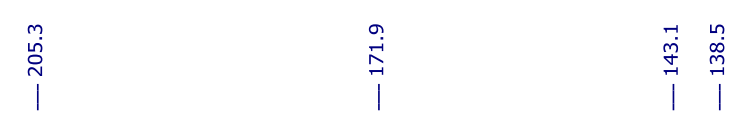

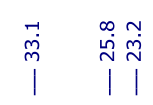

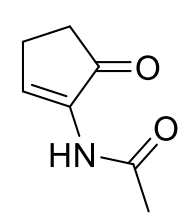

methanol

6

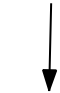

*impurities

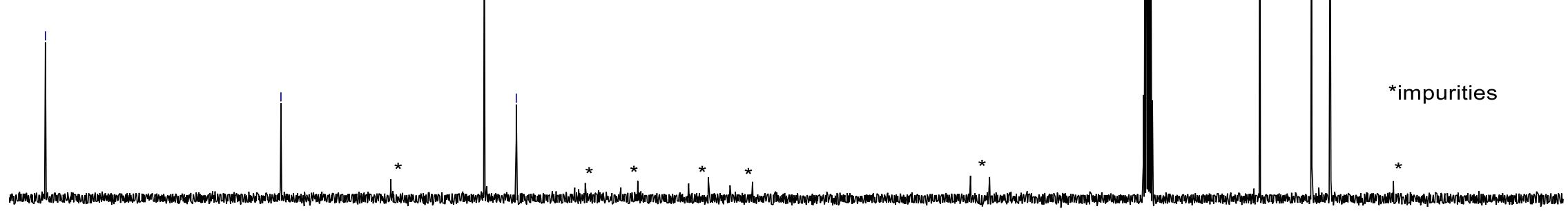

$\begin{array}{llllllllllll}10 & 200 & 190 & 180 & 170 & 160 & 150 & 140 & 130 & 120 & 110 & 100 \\ \text { f1 } & 100 & \end{array}$

$90 \quad 80$

70

60

50

$40 \quad 30$

$20 \quad 10$

0 
$400 \mathrm{MHz}{ }^{1} \mathrm{H}$ NMR spectrum of compound 8 recorded in $\mathrm{CDCl}_{3}$

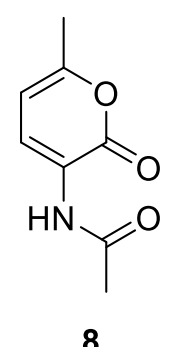

8
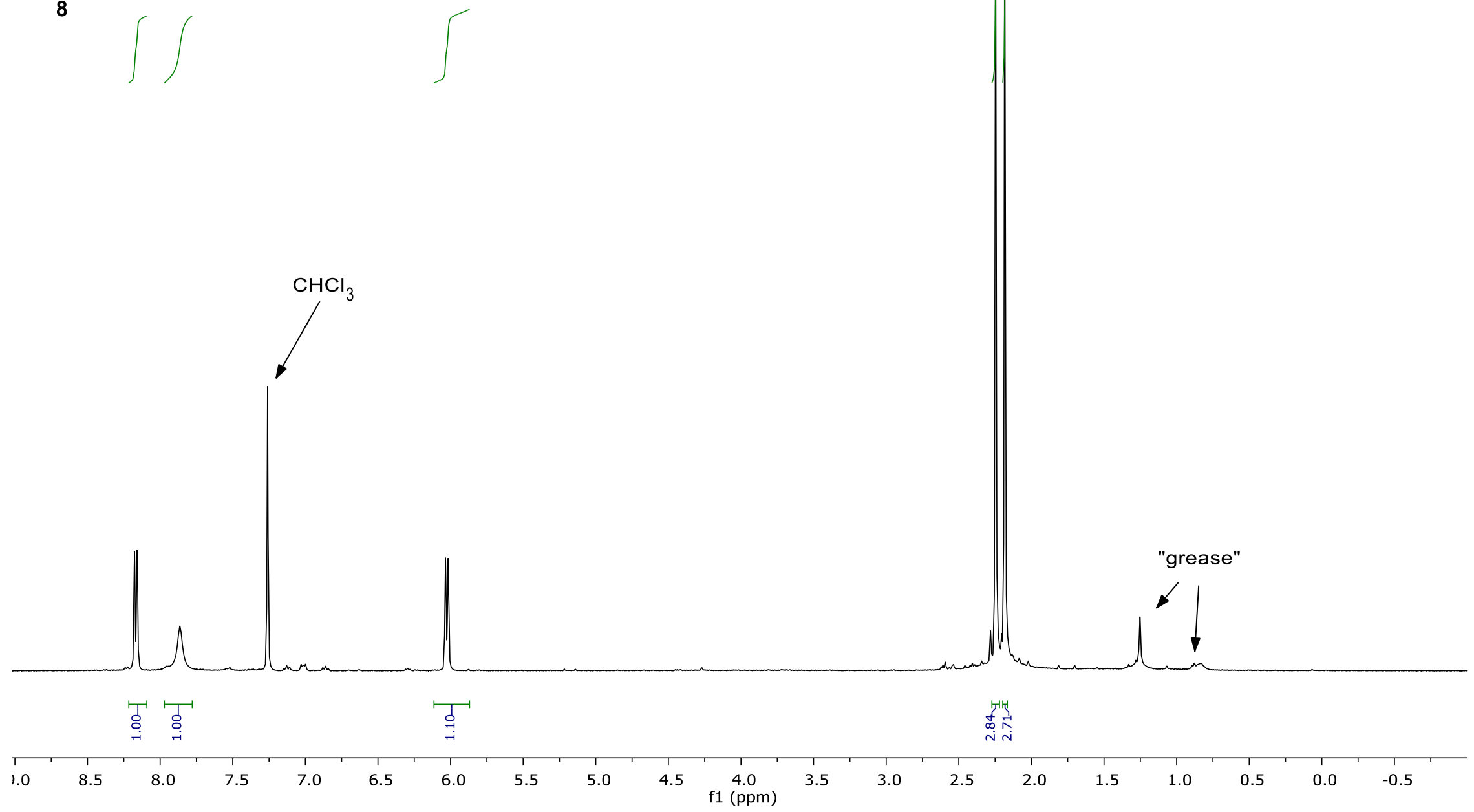
$100 \mathrm{MHz}{ }^{13} \mathrm{C}\left\{{ }^{1} \mathrm{H}\right\}$ NMR spectrum of compound 8 recorded in $\mathrm{CD}_{3} \mathrm{OD}$

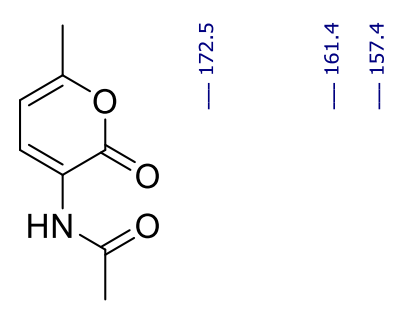

8

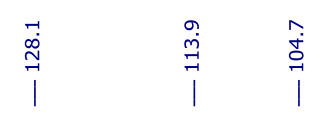

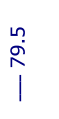

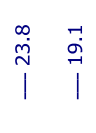

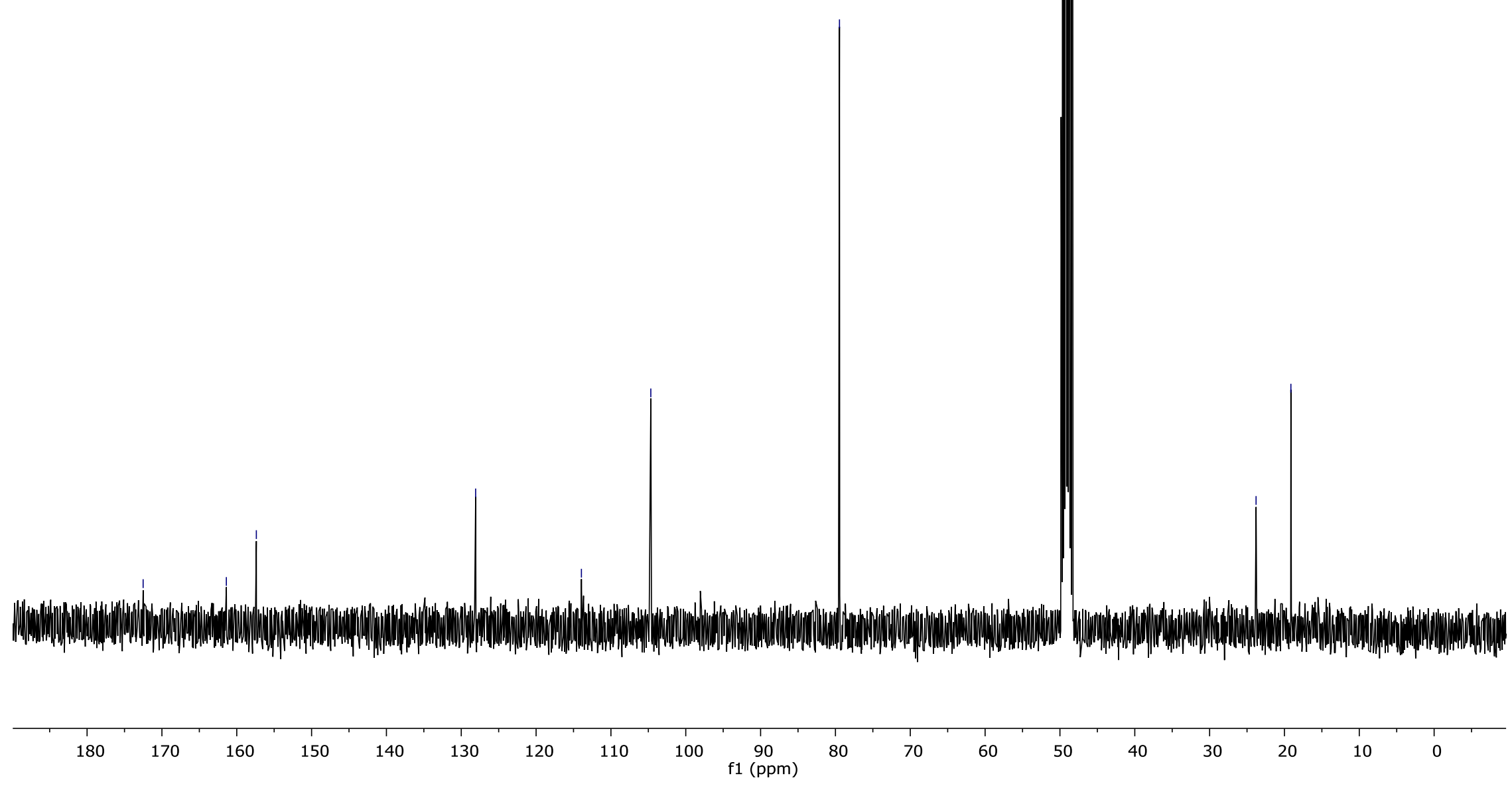

S31 

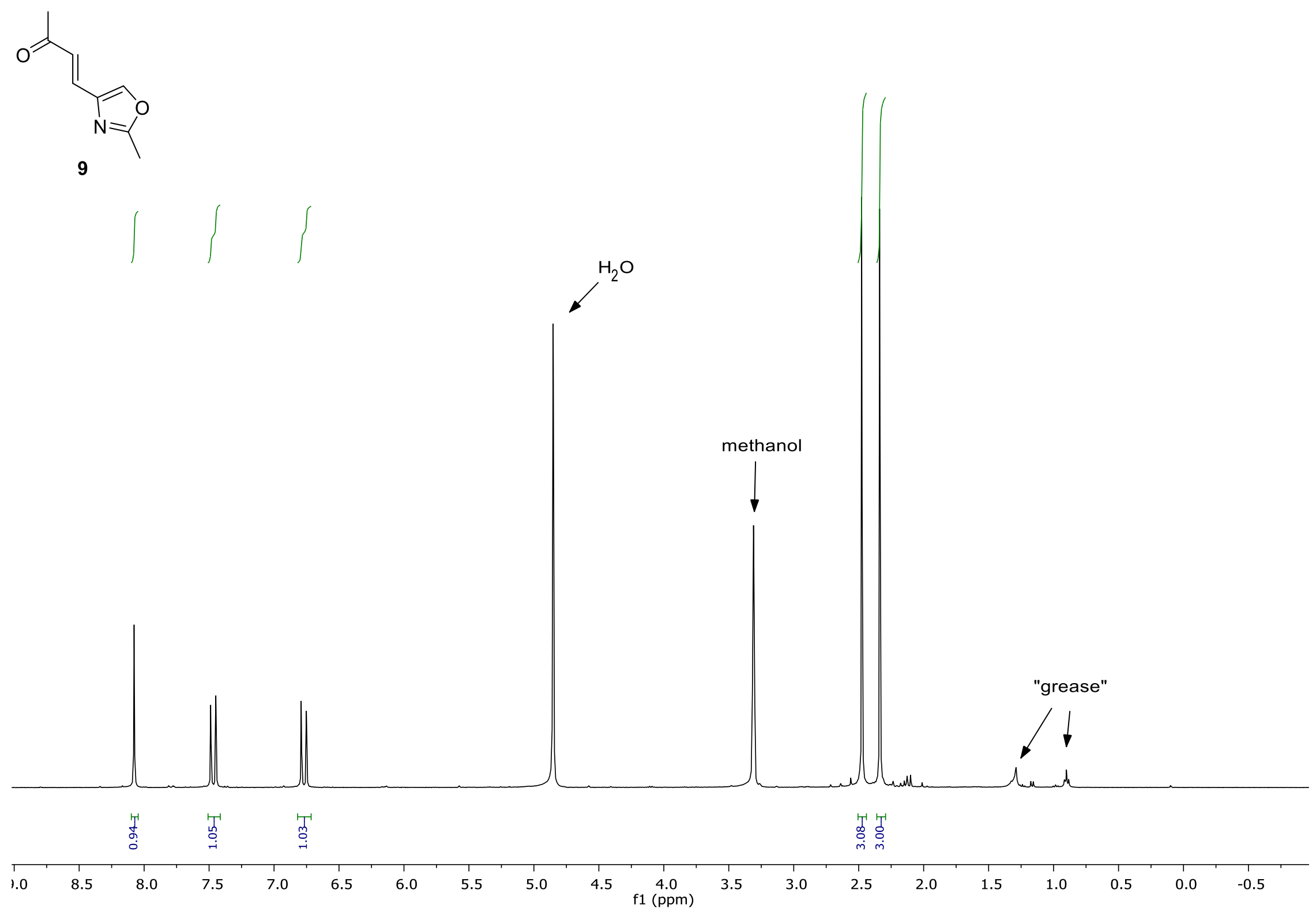
$100 \mathrm{MHz}{ }^{13} \mathrm{C}\left\{{ }^{1} \mathrm{H}\right\} \mathrm{NMR}$ spectrum of compound 9 recorded in $\mathrm{CD}_{3} \mathrm{OD}$
$\stackrel{\infty}{\stackrel{\infty}{\sim}}$

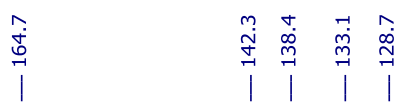
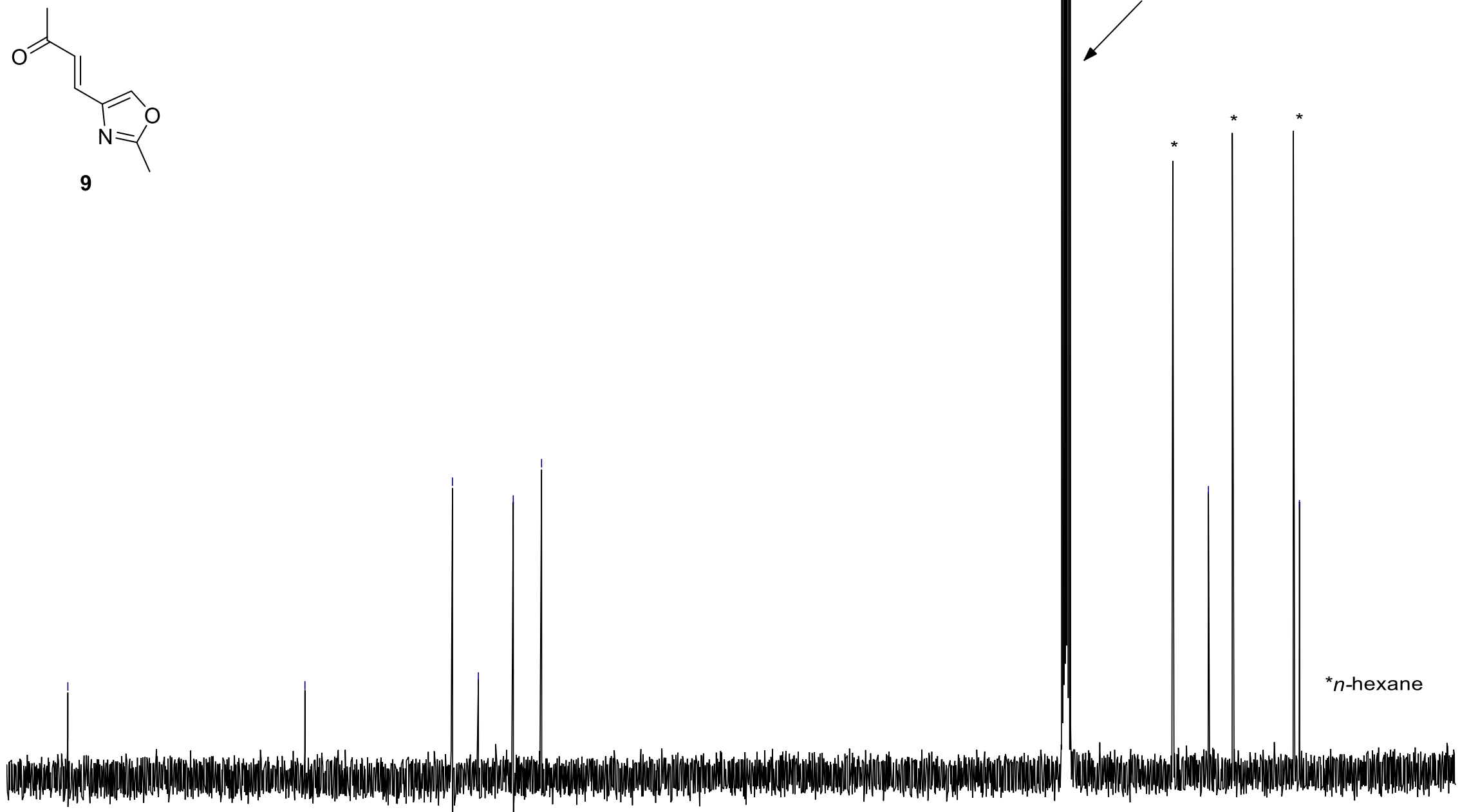

$170 \quad 160$

150

130

11

f1 $\stackrel{100}{(\mathrm{ppm})}$

$90 \quad 80$

50

$40 \quad 30$

20

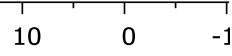


$400 \mathrm{MHz}{ }^{1} \mathrm{H}$ NMR spectrum of compound 10 recorded in $\mathrm{CD}_{3} \mathrm{OD}$

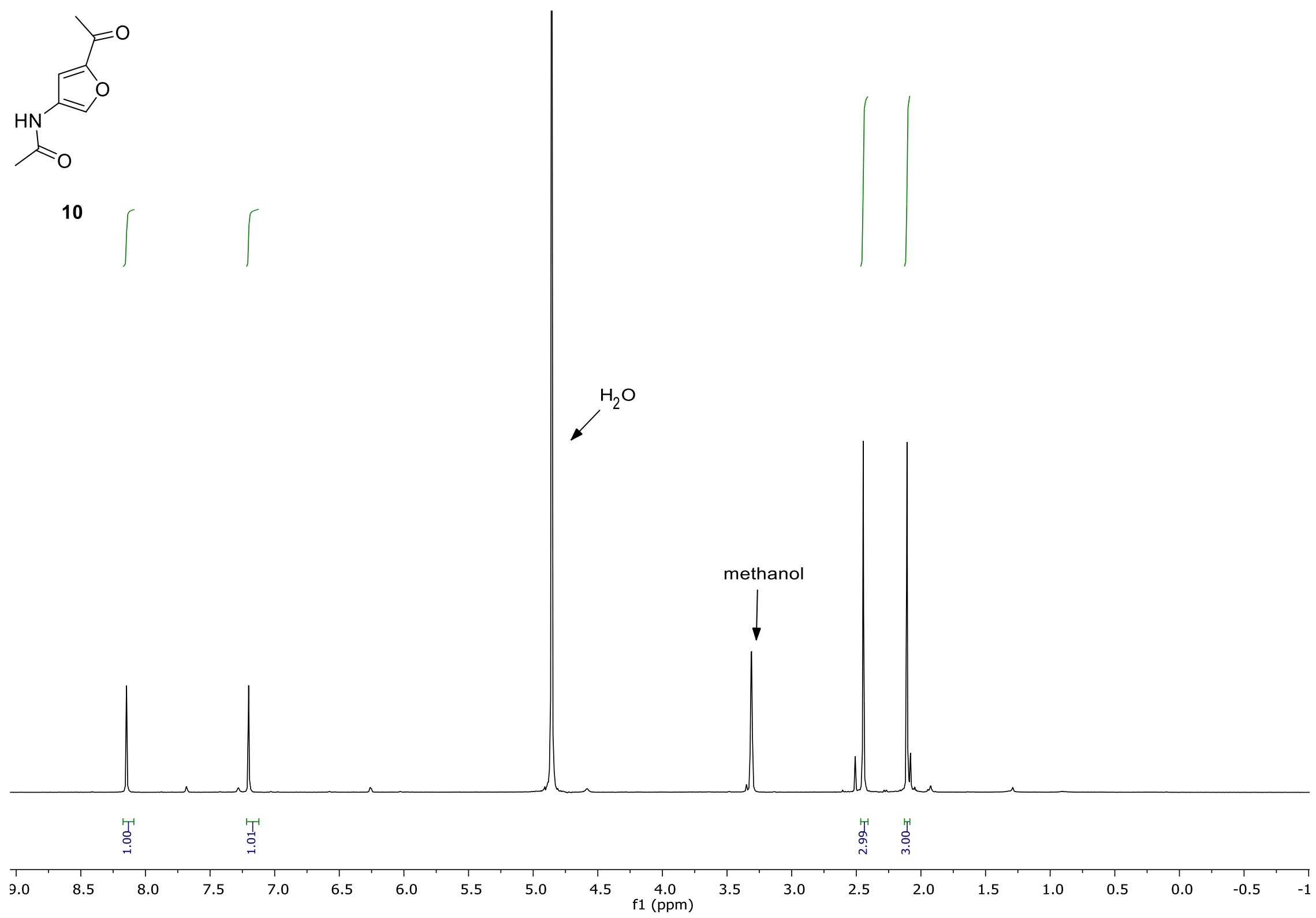


$100 \mathrm{MHz}{ }^{13} \mathrm{C}\left\{{ }^{1} \mathrm{H}\right\}$ NMR spectrum of compound 10 recorded in $\mathrm{CD}_{3} \mathrm{OD}$

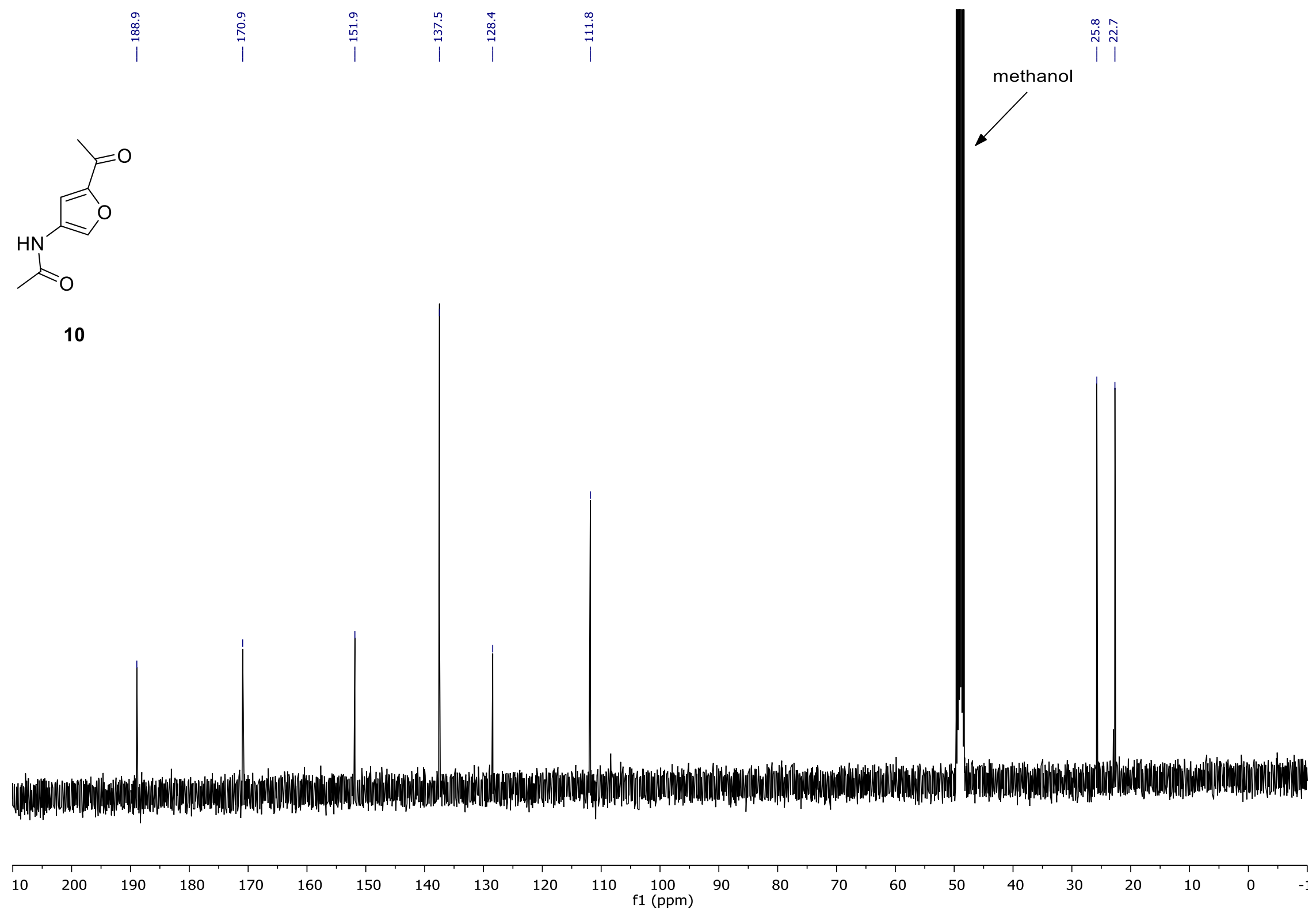


$400 \mathrm{MHz}{ }^{1} \mathrm{H}$ NMR spectrum of compound 11 recorded in $\mathrm{CD}_{3} \mathrm{OD}$

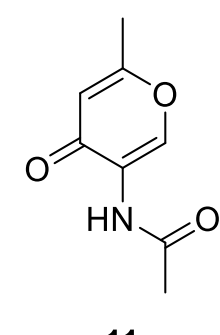

11
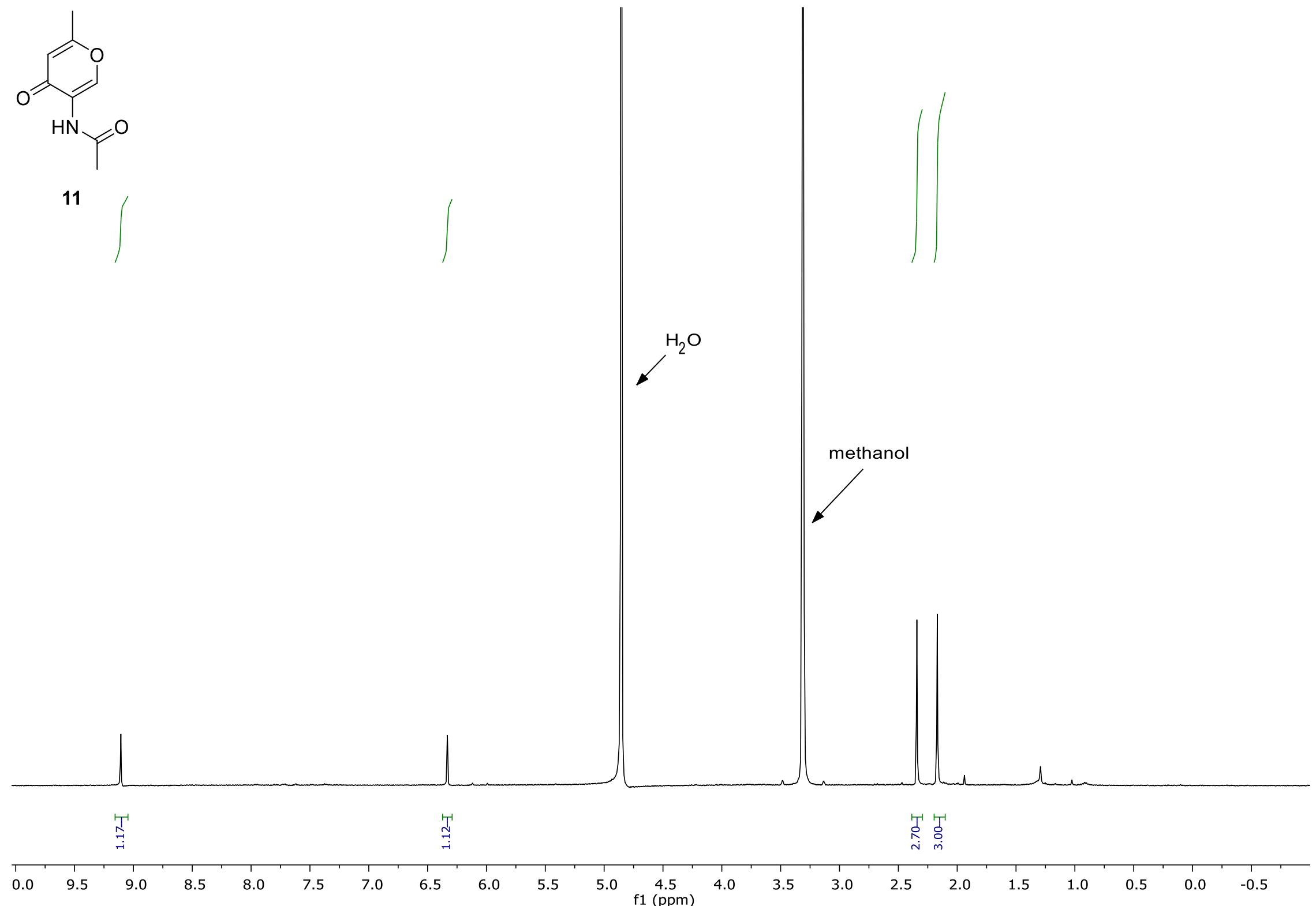
$100 \mathrm{MHz}{ }^{13} \mathrm{C}\left\{{ }^{1} \mathrm{H}\right\}$ NMR spectrum of compound 11 recorded in $\mathrm{CD}_{3} \mathrm{OD}$

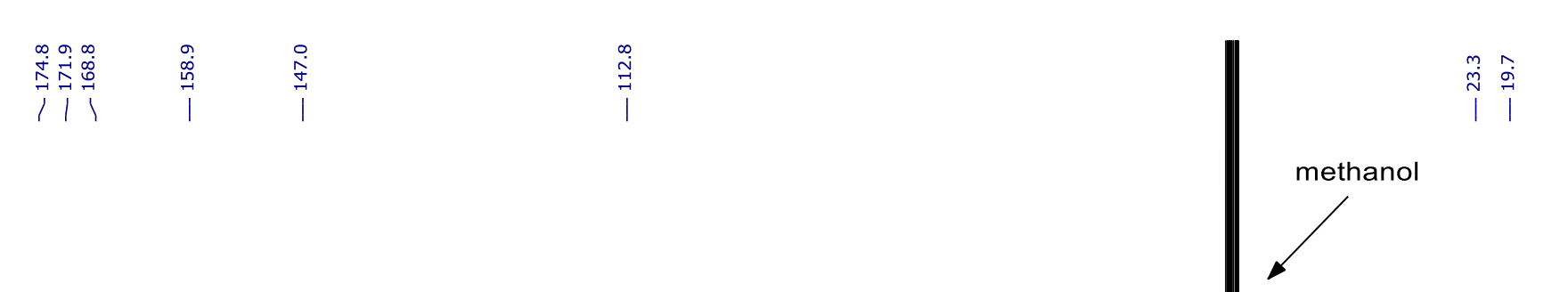

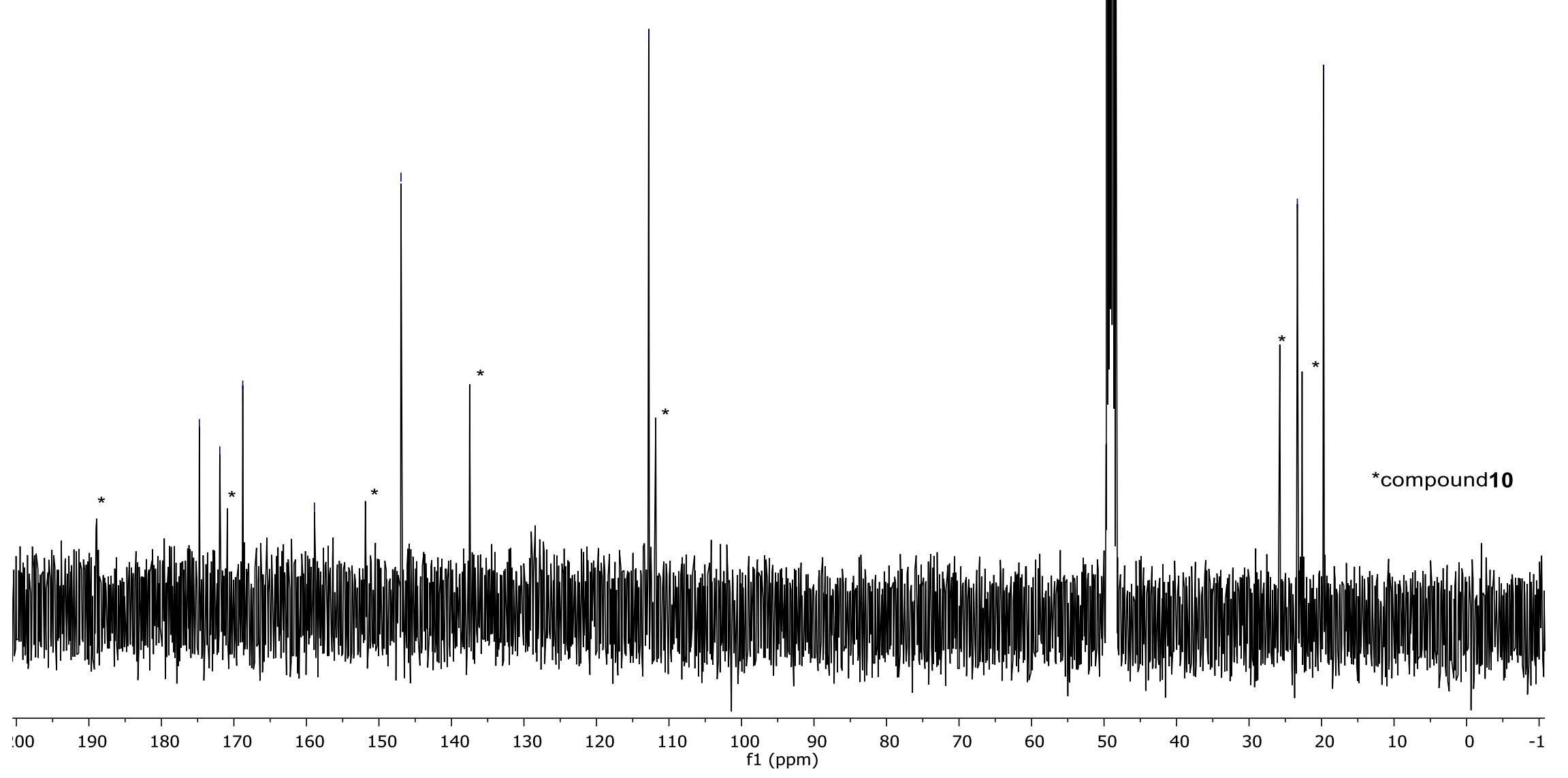


$400 \mathrm{MHz}{ }^{1} \mathrm{H}$ NMR spectrum of compound 12 recorded in $\mathrm{CD}_{3} \mathrm{OD}$

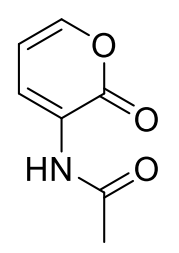

12

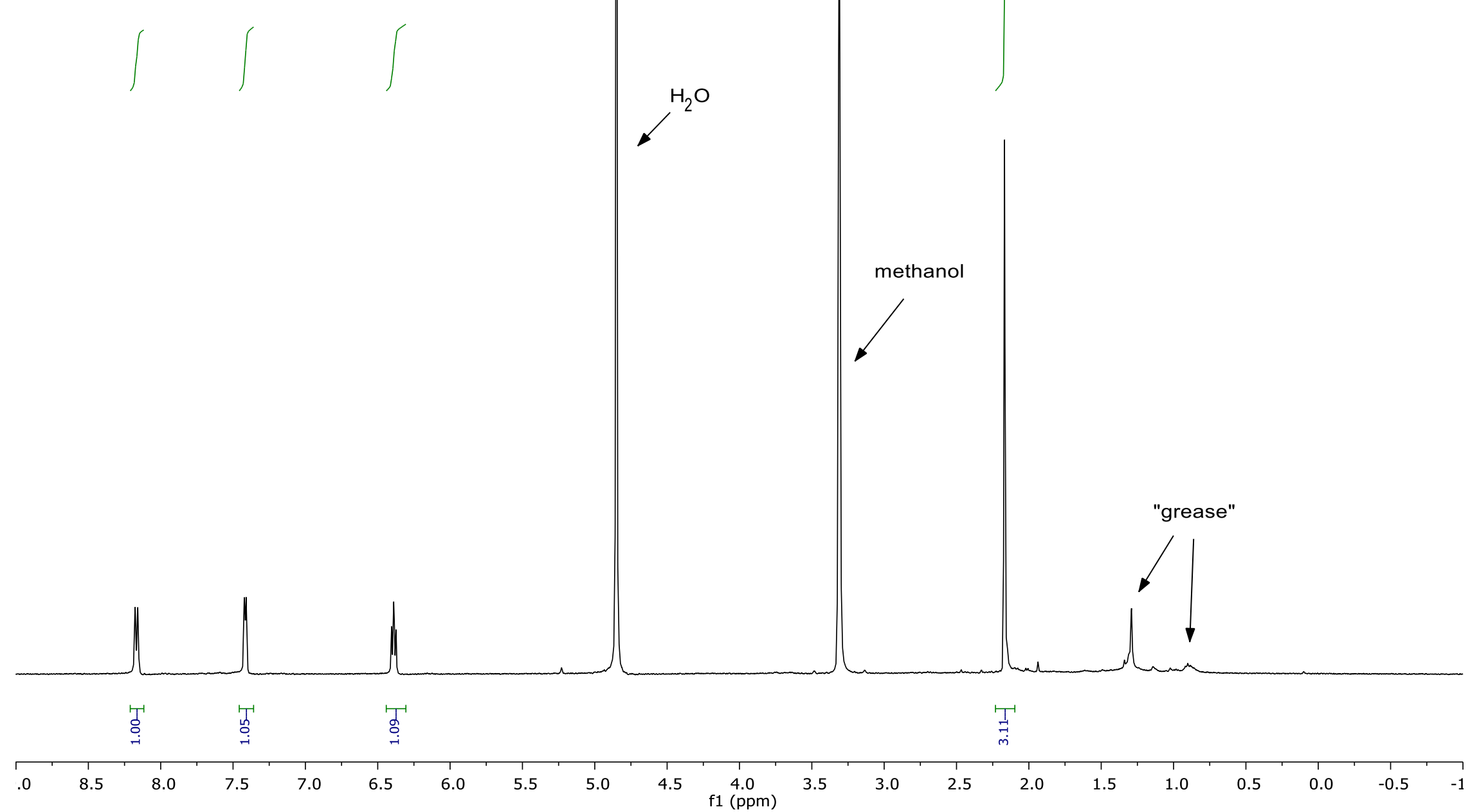


$100 \mathrm{MHz}{ }^{13} \mathrm{C}\left\{{ }^{1} \mathrm{H}\right\}$ NMR spectrum of compound 12 recorded in $\mathrm{CD}_{3} \mathrm{OD}$

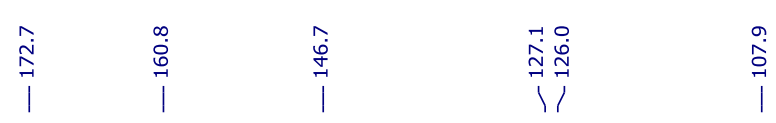

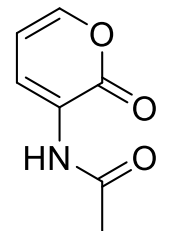

12

methano
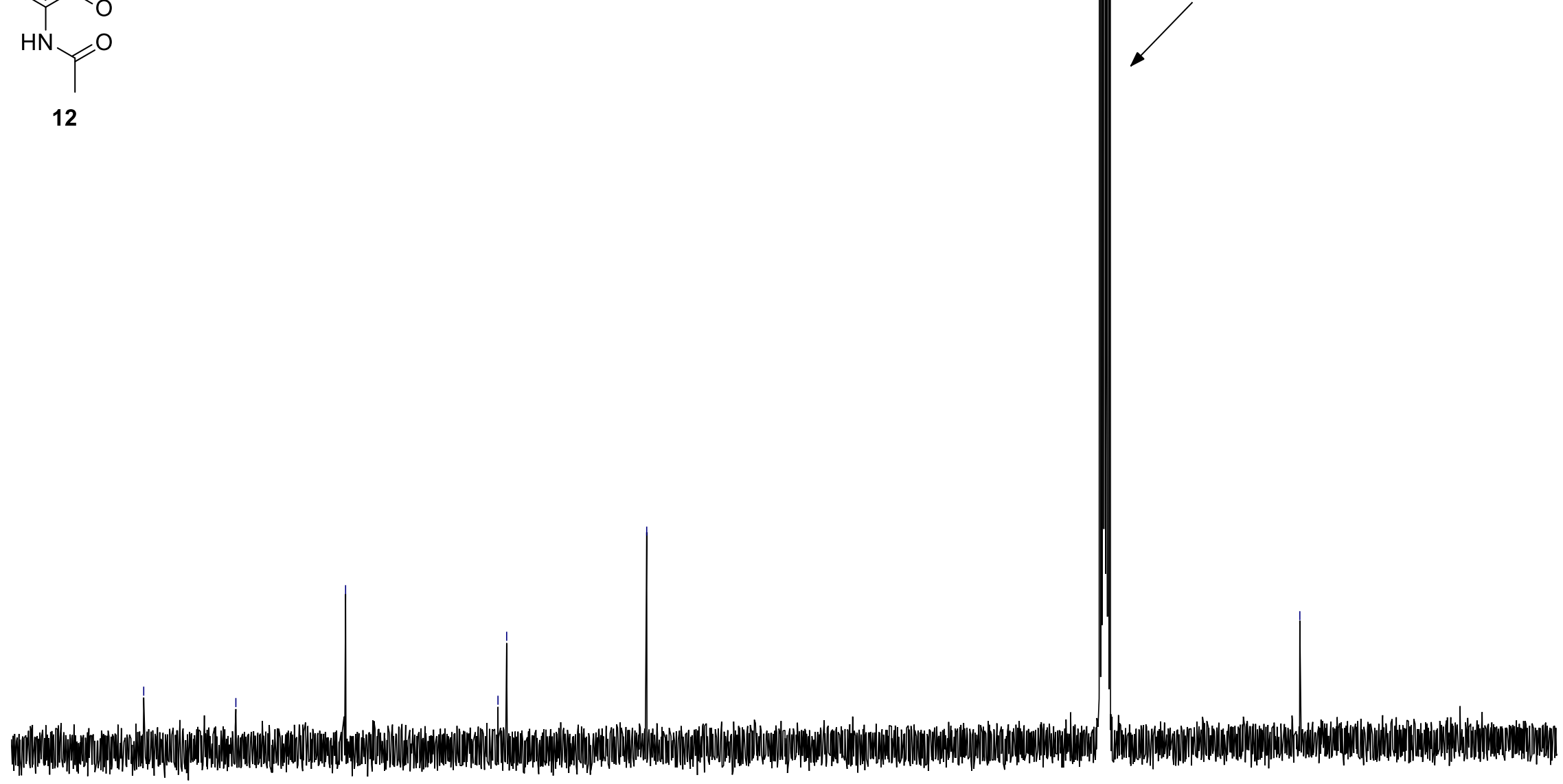

180

170

160

150

140

130

120

110

100

90
$\mathrm{f} 1(\mathrm{ppm})$

$80 \quad 70$

60

50

40

30

20

10 
$400 \mathrm{MHz}{ }^{1} \mathrm{H}$ NMR spectrum of compound 13 recorded in $\mathrm{CD}_{3} \mathrm{OD}$

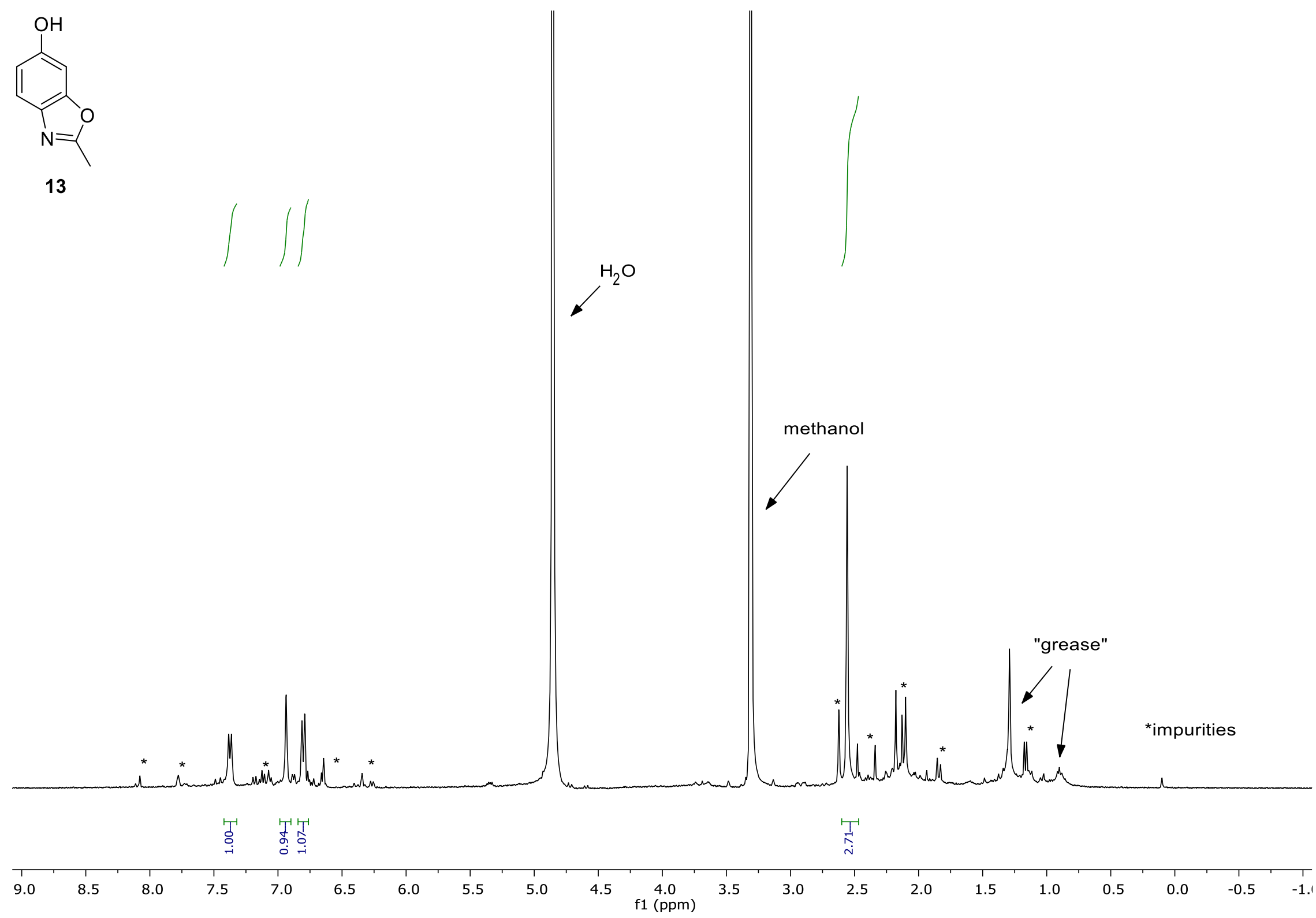


$100 \mathrm{MHz}{ }^{13} \mathrm{C}\left\{{ }^{1} \mathrm{H}\right\}$ NMR spectrum of compound 13 recorded in $\mathrm{CD}_{3} \mathrm{OD}$

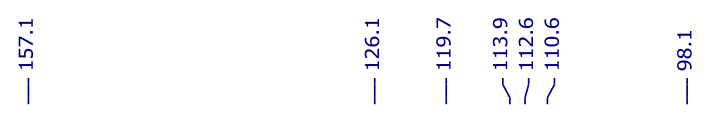

methanol
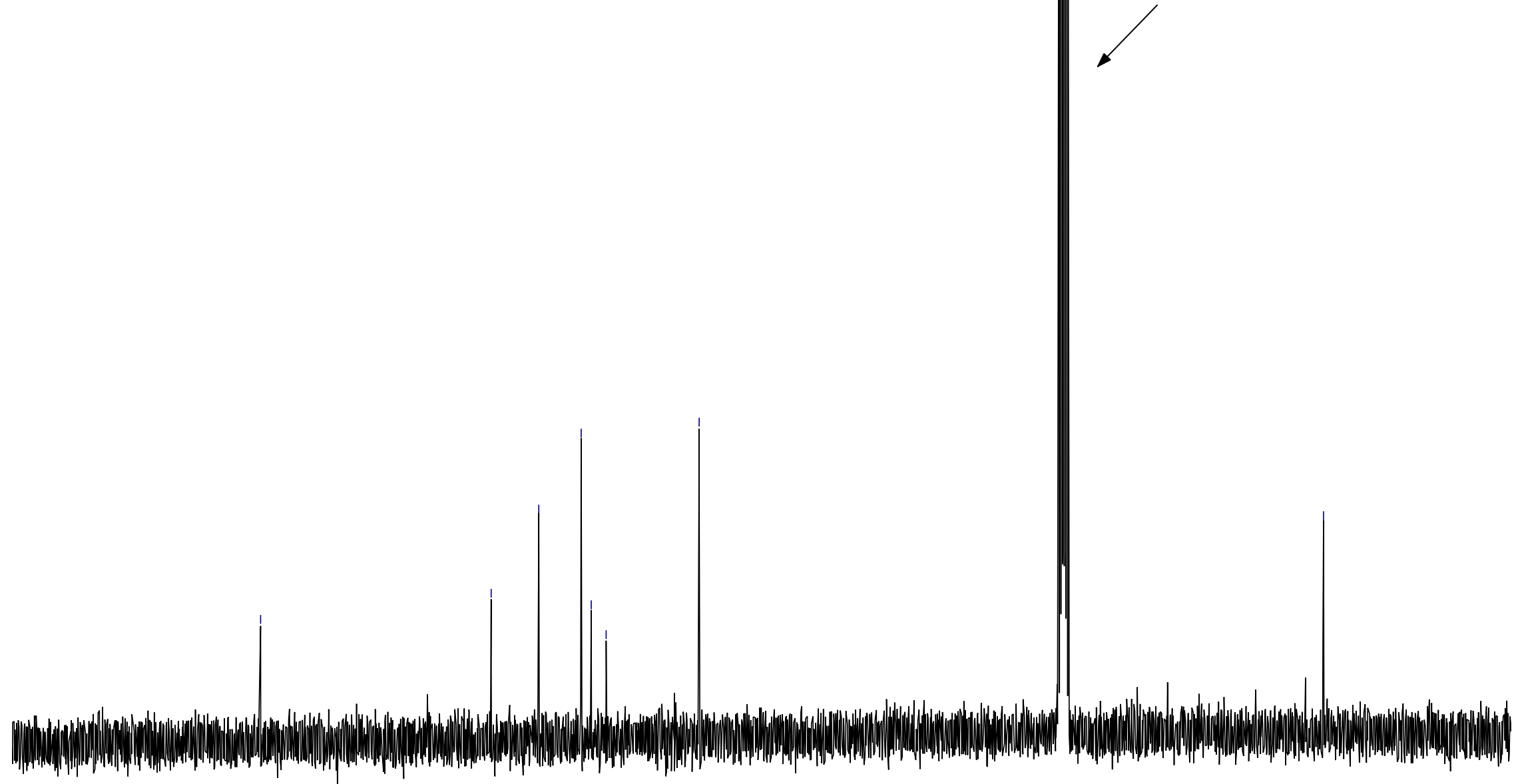

$90 \quad 180$ 
$400 \mathrm{MHz}{ }^{1} \mathrm{H}$ NMR spectrum of compound 15 recorded in $\mathrm{CD}_{3} \mathrm{OD}$
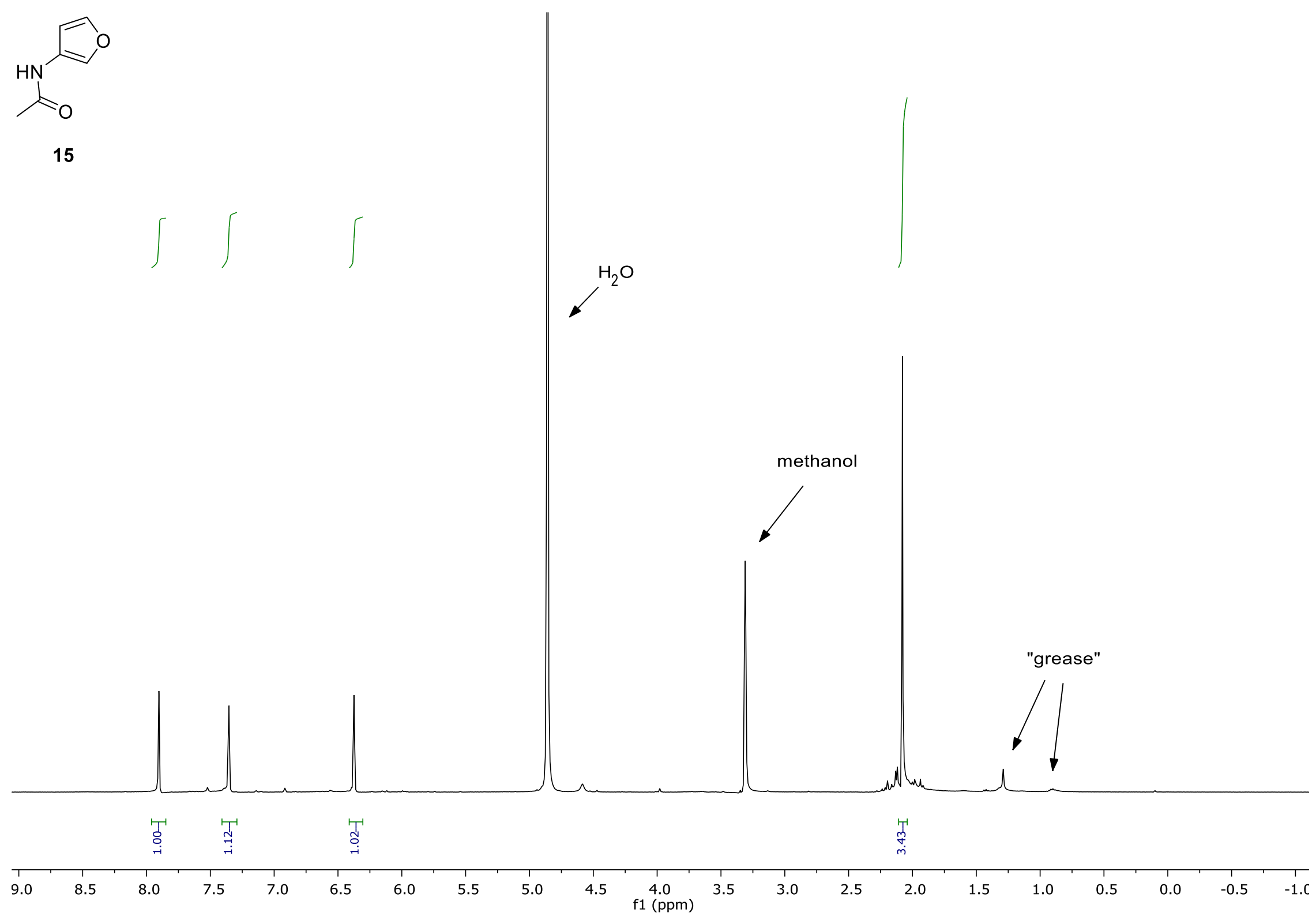
$100 \mathrm{MHz}{ }^{13} \mathrm{C}\left\{{ }^{1} \mathrm{H}\right\}$ NMR spectrum of compound 15 recorded in $\mathrm{CD}_{3} \mathrm{OD}$

\begin{tabular}{|c|c|c|c|}
\hline $\begin{array}{l}\stackrel{0}{0} \\
\stackrel{1}{\mid}\end{array}$ & 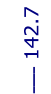 & $\stackrel{\underset{m}{\sigma}}{\stackrel{\sim}{m}}$ & 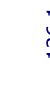 \\
\hline
\end{tabular}

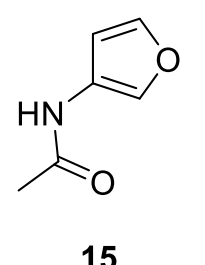

methano

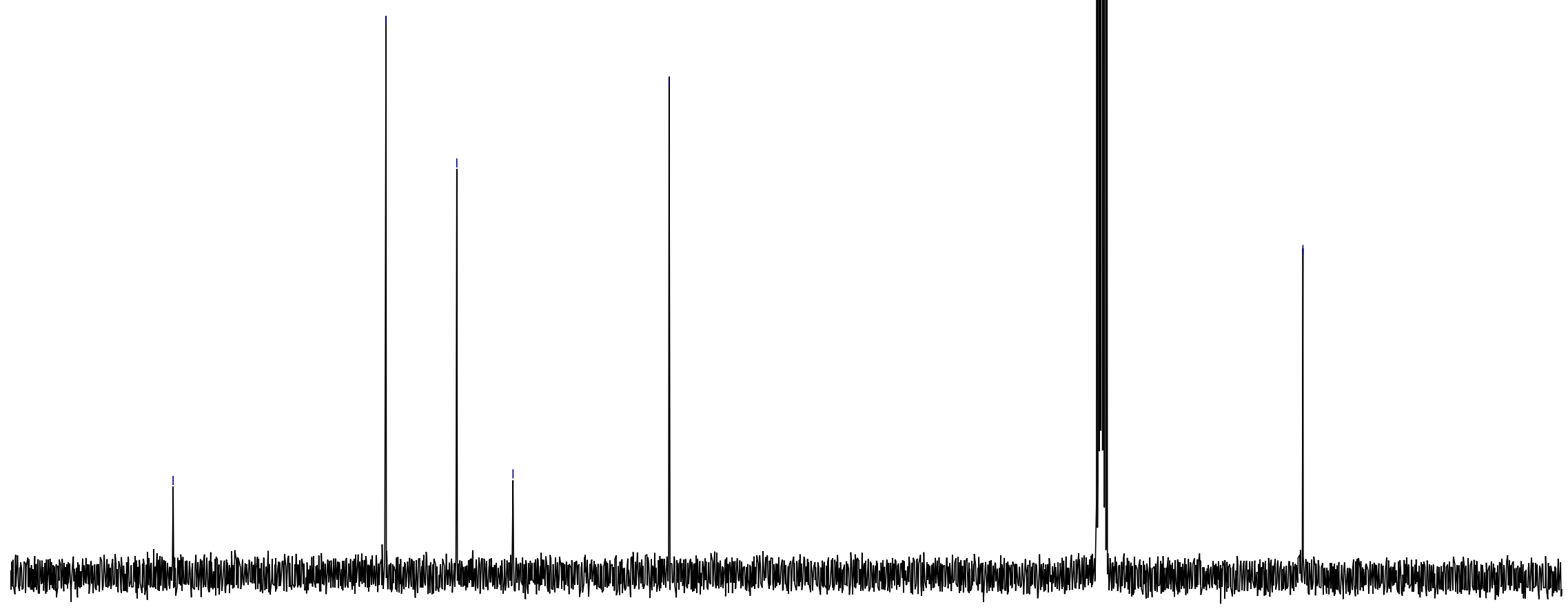

